\title{
Gauge Invariant Spectral Cauchy Characteristic Extraction
}

\author{
Casey J. Handmer ${ }^{1}$, Béla Szilágyi ${ }^{1}$, Jeffrey Winicour ${ }^{2}$ \\ ${ }^{1}$ Theoretical Astrophysics 350-17, California Institute of Technology, Pasadena, \\ California 91125, USA \\ ${ }^{2}$ Department of Physics and Astronomy University of Pittsburgh, Pittsburgh, PA \\ 15260, USA \\ E-mail: chandmer@caltech.edu
}

\begin{abstract}
We present gauge invariant spectral Cauchy characteristic extraction. We compare gravitational waveforms extracted from a head-on black hole merger simulated in two different gauges by two different codes. We show rapid convergence, demonstrating both gauge invariance of the extraction algorithm and consistency between the legacy Pitt null code and the much faster Spectral Einstein Code $(\mathrm{SpEC})$.
\end{abstract}




\section{What is CCE? What is gravitational waveform gauge invariance?}

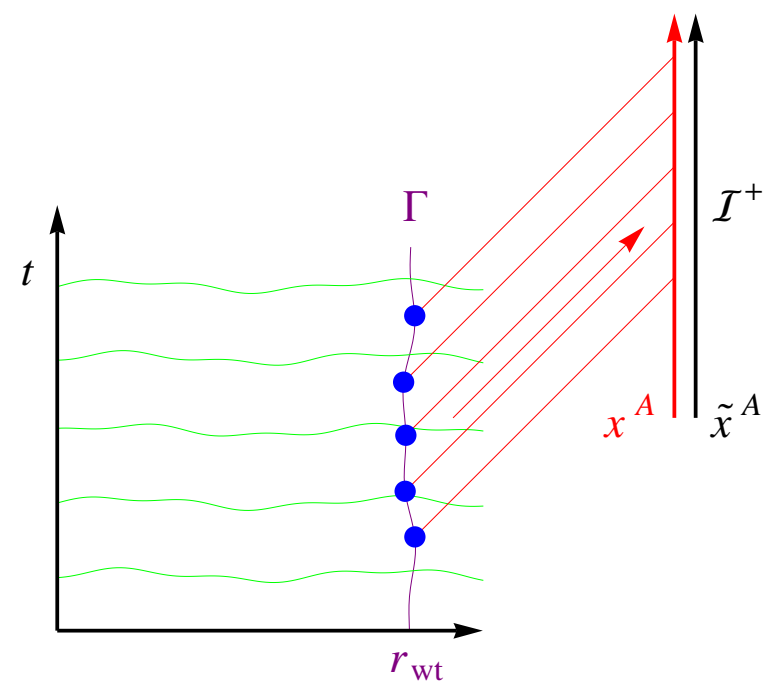

Figure 1: Cauchy characteristic extraction. A Cauchy evolution of the Einstein field equation proceeds on a space-like foliation (green). A finite spheroidal worldtube $\Gamma$ at areal radius $r_{w t}$ forms the inner boundary to a characteristic evolution on a null foliation (red). Based on a coordinate system $x^{A}$ derived from the Cauchy data on the worldtube, gravitational information is propagated to compactified future null infinity $\mathscr{I}^{+}$. At $\mathscr{I}^{+}$an inertial coordinate system $x^{\tilde{A}}$ is co-evolved, in which the desired gauge-invariant waveform can be expressed.

The strong gravitational radiation produced in the inspiral and merger of binary black holes has been a dominant motivation for the construction of gravitational wave observatories. The details of the gravitational waveform supplied by numerical simulation is a key theoretical tool to fully complement the sensitivity of the LIGO, Virgo, GEO, and KAGRA observatories, by enhancing detection and providing useful scientific interpretation of the gravitational signal[1, 2, 3, 4]. Characteristic evolution coupled to Cauchy evolution via Cauchy-characteristic extraction (CCE) provides the most accurate numerical computation of the Bondi news function, which determines both the waveform and the radiated energy and momentum at null infinity. 
In $\mathrm{CCE}$, the Cauchy evolution is used to supply boundary data on a timelike inner worldtube necessary to carry out a characteristic evolution extending to future null infinity $\mathscr{I}^{+}$, where the radiation is computed using the geometric methods developed by Bondi et al.[5], Sachs[6], and Penrose[7], as depicted in Fig. 1. More intuitive methods, including intrinsically inertial compactified hyperboloidal formulations $[8,9$, 10 have not yet found adoption in the evolution of binary black holes. CCE is an initial-boundary value problem based upon a timelike worldtube[11]. It has been implemented as a characteristic evolution code, the Pitt null code [12, 13, which incorporates a Penrose compactification of the spacetime, and which has subsequently been extended to higher order methods by Reisswig et al. [14]. It has more recently been implemented as a spectral code within the Spectral Einstein Code (SpEC) by Handmer and Szilágyi[15], upon which the present work is based.

One technical complication introduced by $\mathrm{CCE}$ is that the coordinates induced on $\mathscr{I}^{+}$by the computational Cauchy coordinates on the inner worldtube do not correspond to inertial observers, i.e., to the coordinates intrinsic to a distant freely falling and non-rotating observatory. The gravitational waveform first obtained in the "computational coordinates" of CCE is in a scrambled form. This gauge ambiguity in the waveform is removed by constructing the transformation between computational coordinates and inertial coordinates at $\mathscr{I}^{+}$. There still remains the freedom in the choice of inertial observers. In special relativistic theories, this freedom is reduced to the translations and Lorentz transformations of the Poincaré group. As explained in Sec. 3, in an asymptotically flat space time the corresponding asymptotic symmetry group consists of supertranslations and Lorentz transformations. This freedom governs the redshift and initial phase of the waveform.

A physically relevant calculation of the radiation flux must also be referred 
to such inertial coordinates at $\mathscr{I}^{+}$. In this paper, the calculation of the energymomentum flux via the Bondi news function is first carried out in the induced worldtube coordinates and then transformed to the inertial coordinates.

\section{Characteristic Formalism}

The characteristic formalism is based upon a family of outgoing null cones emanating from an inner worldtube and extending to infinity where they foliate $\mathscr{I}^{+}$into spherical slices. We let $u$ label these hypersurfaces, $y^{A}(A=2,3)$ be angular coordinates that label the null rays, and $r$ be a surface area coordinate along the outgoing null cones.

Employing the conventions used in [15], in the resulting $x^{\alpha}=\left(u, r, y^{A}\right)$ coordinates, the metric takes the Bondi-Sachs form

$$
\begin{aligned}
d s^{2}= & -\left(e^{2 \beta}(r W+1)-r^{2} h_{A B} U^{A} U^{B}\right) d u^{2} \\
& -2 e^{2 \beta} d u d r-2 r^{2} h_{A B} U^{B} d u d y^{A}+r^{2} h_{A B} d y^{A} d y^{B},
\end{aligned}
$$

where $h^{A B} h_{B C}=\delta_{C}^{A}$ and $\operatorname{det}\left(h_{A B}\right)=\operatorname{det}\left(q_{A B}\right)$, with $q_{A B}$ a unit sphere metric. In analyzing the Einstein equations, we also use the intermediate variable

$$
Q_{A}=r^{2} e^{-2 \beta} h_{A B} U_{, r}^{B}
$$

The metric coefficients $W, h_{A B}, U^{A}, Q_{A}, \beta$ represent respectively the mass aspect, the spherical 2-metric, the shift and its radial derivative, and the lapse. The vector and tensor fields $h_{A B}, U^{A}, Q_{A}$ are expressed as spin-weighted fields by contracting them with the complex dyad $q^{A}$ for the unit sphere metric satisfying $q^{A} q_{A}=0$, $q^{A} \bar{q}_{A}=2, q^{A}=q^{A B} q_{B}$, with $q^{A B} q_{B C}=\delta_{C}^{A}$ and $q_{A B}=\left(q_{A} \bar{q}_{B}+\bar{q}_{A} q_{B}\right) / 2$. Under this convention, the spin-weighted functions $U=U^{A} q_{A}$ and $Q=Q_{A} q^{A}$, while $J=h_{A B} q^{A} q^{B} / 2$ uniquely determines the spherical 2-metric component of the general 4-metric [13]. We chose a dyad consistent with the computational formulation of the 
spin-weight raising ð operator [16], given by $q^{A}=(-1,-i / \sin \theta)$ in standard spherical coordinates $(\theta, \phi)$. This is regular everywhere except the poles, which we can avoid through careful choice of grid points. It is worth noting that any choice of angular coordinates are possible. Other conventions use multiple patches to avoid singularities at the poles.

A key feature of the Bondi-Sachs formulation is that the Einstein equations can be integrated along the outgoing characteristics in a sequential order. We use a form which first appeared in [17] and was implemented as the Pitt code in [13, 18]:

$$
\begin{aligned}
& \beta, r=N_{\beta}, \\
& \left(r^{2} Q\right)_{, r}=-r^{2}(\bar{\jmath} J+ð K){ }_{r}+2 r^{4} \precsim\left(r^{-2} \beta\right),_{r}+N_{Q}, \\
& U_{, r}=r^{-2} e^{2 \beta} Q+N_{U}, \\
& \left(r^{2} W\right){ }_{r}=\frac{1}{2} e^{2 \beta} \mathcal{R}-1-e^{\beta} \text { ð } \bar{\varnothing} e^{\beta}+\frac{1}{4} r^{-2}\left(r^{4}(ð \bar{U}+\bar{\varnothing} U){ }_{, r}+N_{W},\right.
\end{aligned}
$$$$
\text { and the evolution equation }
$$$$
2(r J)_{, u r}=\left((1+r W)(r J),_{r}\right),_{r}-r^{-1}\left(r^{2} \varpi U\right),_{r}+2 r^{-1} e^{\beta} \check{\partial}^{2} e^{\beta}-(r W)_{r} J+N_{J},
$$

where

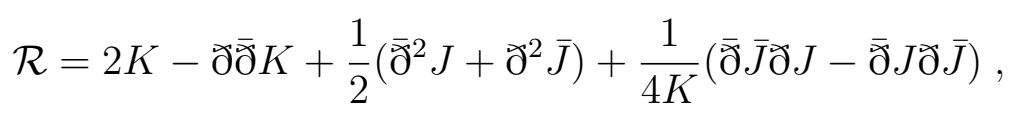

is the curvature scalar associated with $h_{A B}, K^{2}=1+J \bar{J}$ and $N_{\beta}, N_{Q}, N_{W}, N_{J}$ are nonlinear terms given in [13].

On each constant $u$ hypersurface of the spacetime foliation, these equations are integrated in turn. Given $J, \beta$ is solved, then $U, Q$, and $W$ in turn, enabling the computation of $J_{u} . J_{u}$ permits a step forward in time and $J$ is thus defined on the next hypersurface. The radial compactification of infinity is given by

$$
r=r_{w t} \rho /(1-\rho), \quad \frac{1}{2} \leq \rho \leq 1
$$


where the compactification parameter $r_{w t}\left(u, y^{A}\right)$ is the (not necessarily constant) areal radius coordinate on the worldtube.

Angular derivatives are implemented using the action of the operator on spinweighted spherical harmonics, e.g., $\partial U=q^{A} q^{B} U_{A: B}$, where a colon denotes the covariant derivative with respect to $q_{A B}$ [16]. In spherical coordinates, this takes the explicit form for a spin-weight-s field $\eta$

$$
\begin{aligned}
& \gtrsim \eta=-\left(\sin ^{s} \theta\right)\left(\frac{\partial}{\partial \theta}+\frac{i}{\sin \theta} \frac{\partial}{\partial \phi}\right)\left(\sin ^{-s} \theta \eta\right) \\
& \bar{\partial} \eta=-\left(\sin ^{-s} \theta\right)\left(\frac{\partial}{\partial \theta}-\frac{i}{\sin \theta} \frac{\partial}{\partial \phi}\right)\left(\sin ^{s} \theta \eta\right)
\end{aligned}
$$

$\bar{\delta}$ is the associated spin-weight lowering operator.

The spectral algorithm used to solve these equations and the treatment of the nonlinear terms $N_{\beta}, N_{Q}, N_{U}, N_{W}, N_{J}$ are detailed in Handmer and Szilágyi[15]. Here, we extend the characteristic spectral algorithm to calculating the gauge invariant Bondi news at $\mathscr{I}^{+}$.

\section{Waveforms at $\mathscr{I}^{+}$}

For technical simplicity, the theoretical derivation of the waveform at infinity is best presented in terms of an inverse surface-area coordinate $\ell=1 / r$, where $\ell=0$ at $\mathscr{I}^{+}$. In the resulting $x^{\mu}=\left(u, \ell, x^{A}\right)$ conformal Bondi coordinates, the physical spacetime metric $g_{\mu \nu}$ has the conformal compactification $\hat{g}_{\mu \nu}=\ell^{2} g_{\mu \nu}$, where $\hat{g}_{\mu \nu}$ is smooth at $\mathscr{I}^{+}$and, referring to the metric (2.1), takes the form[11]

$$
\begin{aligned}
\hat{g}_{\mu \nu} d x^{\mu} d x^{\nu}= & -\left(e^{2 \beta}\left(\ell^{2}+\ell W\right)-h_{A B} U^{A} U^{B}\right) d u^{2}+2 e^{2 \beta} d u d \ell \\
& -2 h_{A B} U^{B} d u d x^{A}+h_{A B} d x^{A} d x^{B} .
\end{aligned}
$$

As described in [19, 20], both the Bondi news function $N\left(u, x^{A}\right)$ and the Newman- 
Penrose Weyl tensor component [21]

$$
\Psi_{4}^{0}\left(u, x^{A}\right)=\lim _{r \rightarrow \infty} r \psi_{4}
$$

which describe the waveform, are determined by the asymptotic limit at $\mathscr{I}^{+}$of the tensor field

$$
\hat{\Sigma}_{\mu \nu}=\frac{1}{\ell}\left(\hat{\nabla}_{\mu} \hat{\nabla}_{\nu}-\frac{1}{4} \hat{g}_{\mu \nu} \hat{\nabla}^{\alpha} \hat{\nabla}_{\alpha}\right) \ell
$$

This limit is constructed from the leading coefficients in an expansion of the metric about $\mathscr{I}^{+}$in powers of $\ell$. We thus write

$$
h_{A B}=H_{A B}+\ell c_{A B}+O\left(\ell^{2}\right) .
$$

Conditions on the asymptotic expansion of the remaining components of the metric follow from the Einstein equations:

$$
\begin{gathered}
\beta=H+O\left(\ell^{2}\right), \\
U^{A}=L^{A}+2 \ell e^{2 H} H^{A B} D_{B} H+O\left(\ell^{2}\right),
\end{gathered}
$$

and

$$
W=D_{A} L^{A}+\ell\left(e^{2 H} \mathcal{R} / 2+D_{A} D^{A} e^{2 H}-1\right)+O\left(\ell^{2}\right),
$$

where $H$ and $L$ are the asymptotic limits of $\beta$ and $U$ and where $\mathcal{R}$ and $D_{A}$ are the 2-dimensional curvature scalar and covariant derivative associated with $H_{A B}$.

The expansion coefficients $H, H_{A B}, c_{A B}$, and $L^{A}$ (all functions of $u$ and $x^{A}$ ) completely determine the radiation field. One can further specialize the Bondi coordinates to be inertial at $\mathscr{I}^{+}$, i.e., have Minkowski form, in which case $H=$ $L^{A}=0, H_{A B}=q_{A B}$ (the unit sphere metric) so that the radiation field is completely determined by $c_{A B}$. However, the characteristic extraction of the waveform is carried out in computational coordinates (determined by the Cauchy data on the extraction worldtube) so this inertial simplification cannot be assumed. 
In order to first compute the Bondi news function in the $\hat{g}_{\mu \nu}$ computational frame, it is necessary to determine the conformal factor $\omega$ relating $H_{A B}$ to a unit sphere metric $Q_{A B}$, i.e., to an inertial conformal Bondi frame[11] satisfying

$$
Q_{A B}=\omega^{2} H_{A B}
$$

(See 22] for a discussion of how the news in an arbitrary conformal frame is related to its expression in this inertial Bondi frame.) We can determine $\omega$ by solving the elliptic equation governing the conformal transformation of the curvature scalar (2.8) to a unit sphere geometry:

$$
\mathcal{R}=2\left(\omega^{2}+H^{A B} D_{A} D_{B} \log \omega\right)
$$

The elliptic equation (3.9) need only be solved at the initial time where, with initial data $\left.J\right|_{\mathscr{I}+}=0, H^{A B} D_{A} D_{B}$ simplifies to the 2-Laplacian on the unit sphere. Then, as described in the next section, application of the Einstein equations on $\mathscr{I}^{+}$determines the time dependence of $\omega$ according to

$$
2 \hat{n}^{\alpha} \partial_{\alpha} \log \omega=-e^{-2 H} D_{A} L^{A},
$$

where $\hat{n}^{\alpha}=\hat{g}^{\alpha \beta} \nabla_{\beta} \ell$ is the null vector tangent to the generators of $\mathscr{I}^{+}$. We use (3.10) to evolve $\omega$ along the generators of $\mathscr{I}^{+}$given a solution of $(3.9)$ as initial condition.

First recall some basic elements of Penrose compactification. In a general conformal frame with metric $\hat{g}_{\mu \nu}=\Omega^{2} g_{\mu \nu}$, where $\Omega=0$ on $\mathscr{I}^{+}$, the vacuum Einstein equations $G_{\mu \nu}=0$ take the form

$$
\Omega^{2} \hat{G}_{\mu \nu}+2 \Omega \hat{\nabla}_{\mu} \hat{\nabla}_{\nu} \Omega-\hat{g}_{\mu \nu}\left(2 \Omega \hat{\nabla}^{\rho} \hat{\nabla}_{\rho} \Omega-3\left(\hat{\nabla}^{\rho} \Omega \hat{\nabla}_{\rho} \Omega\right)\right)=0 .
$$

It immediately follows that

$$
\left.\left(\hat{\nabla}^{\rho} \Omega\right) \hat{\nabla}_{\rho} \Omega\right|_{\mathscr{I}^{+}}=0
$$


so that $\mathscr{I}^{+}$is a null hypersurface and that

$$
\left.\left[\hat{\nabla}_{\mu} \hat{\nabla}_{\nu} \Omega-\frac{1}{4} \hat{g}_{\mu \nu} \hat{\nabla}^{\rho} \hat{\nabla}_{\rho} \Omega\right]\right|_{\mathscr{I}^{+}}=0
$$

With respect to this frame, the construction of an inertial conformal frame proceeds as follows. We introduce a new conformal factor $\tilde{\Omega}=\omega \Omega$, with $\tilde{g}_{\mu \nu}=\omega^{2} \hat{g}_{\mu \nu}$ by requiring, in accord with 3.10 ,

$$
\left.\left[2 \hat{n}^{\sigma} \partial_{\sigma} \omega+\omega \hat{\nabla}_{\sigma} \hat{n}^{\sigma}\right]\right|_{\mathscr{I}+}=0, \quad \hat{n}^{\sigma}=\hat{g}^{\rho \sigma} \nabla_{\rho} \Omega
$$

As a result, it follows from a straightforward calculation that

$$
\tilde{\nabla}^{\rho} \tilde{\nabla}_{\rho} \tilde{\Omega}_{\mathscr{I}_{+}}=0
$$

i.e., in the $\tilde{g}_{\mu \nu}$ conformal frame $\mathscr{I}^{+}$is null, shear- and divergence-free.

It also follows that

$$
\left.\tilde{n}^{\sigma} \tilde{\nabla}_{\sigma} \tilde{n}^{\nu}\right|_{\mathscr{I}+}=0
$$

where $\tilde{n}^{\sigma}=\tilde{g}^{\rho \sigma} \tilde{\nabla}_{\rho} \tilde{\Omega}$, i.e., in the $\tilde{g}_{\mu \nu}$ frame, $\tilde{n}^{\sigma}$ is an affinely parametrized null generator of $\mathscr{I}^{+}$.

To construct inertial coordinates $\left(\tilde{u}, x^{\tilde{A}}\right)$ on $\mathscr{I}^{+}$, we first assign angular coordinates $x^{\tilde{A}}$ to each point of the initial spacelike spherical slice $u=u_{0}$ of $\mathscr{I}^{+}$. We then propagate these coordinates along the generators of $\mathscr{I}^{+}$according to

$$
\left.\tilde{n}^{\rho} \partial_{\rho} x^{\tilde{A}}\right|_{\mathscr{I}+}=\left.\omega^{-1} \hat{n}^{\rho} \partial_{\rho} x^{\tilde{A}}\right|_{\mathscr{I}+}=0
$$

In addition, we require

$$
\left.\tilde{n}^{\rho} \partial_{\rho} \tilde{u}\right|_{\mathscr{I}+}=\left.\omega^{-1} \hat{n}^{\rho} \partial_{\rho} \tilde{u}\right|_{\mathscr{I}+}=1
$$

so that $\tilde{u}$ is an affine parameter along the generators in the $\tilde{g}_{\mu \nu}$ conformal frame. 


\section{News}

The Bondi news function $N$ is computed in the computational coordinates with the appropriate conformal transformation. It is then interpolated onto the inertial coordinates. The formalism follows that of [13], Appendix B (with a sign error in $s_{3}$ corrected):

$$
N=\frac{1}{4 \omega A}\left(s_{1}+s_{2}+\frac{1}{4}(\check{\bar{U}}+\overline{\widetilde{ }} U) s_{3}-4 \omega^{-2} s_{4}+2 \omega^{-1} s_{5}\right),
$$


where $A=\omega e^{2 \beta}$ and the $s_{i}$ terms are

$$
\begin{aligned}
& s_{1}=\left(J^{2} \bar{J}_{, \ell u}+J \bar{J} J_{\ell \ell u}-2 J K K_{, \ell u}-2 J K_{, \ell u}+2 J_{, \ell u} K+2 J_{, \ell u}\right) /(K+1), \\
& s_{2}=\left(ð J_{, \ell} J \bar{J} \bar{U}+2 ð J_{, \ell} K \bar{U}+2 ð J_{, \ell} \bar{U}+ð \bar{J}_{, \ell} J^{2} \bar{U}\right. \\
& -2 ð K_{, \ell} J K \bar{U}-2 ð K_{, \ell} J \bar{U}+2 ð U J \bar{J} K_{, \ell}-2 ð U J \bar{J}_{, \ell} K \\
& -2 ð U J \bar{J}_{, \ell}+4 ð U K K_{, \ell}+4 ð U K_{, \ell}+2 ð \bar{U} J \bar{J} J_{, \ell}-2 ð \bar{U} J K K_{, \ell} \\
& -2 ð \bar{U} J K_{, \ell}+4 ð \bar{\mho} J_{, \ell} K+4 ð \bar{U} J_{, \ell}+\bar{\partial} J_{\ell} J \bar{J} U+2 \bar{\varnothing} J_{, \ell} K U+2 \bar{ð} J_{, \ell} U \\
& +\bar{\varnothing} \bar{J}_{, \ell} J^{2} U-2 \bar{\varnothing} K_{, \ell} J K U-2 \bar{\varnothing} K_{, \ell} J U+2 \bar{\varnothing} U J^{2} \bar{J}_{, \ell}-2 \bar{\varnothing} U J K K_{, \ell}
\end{aligned}
$$

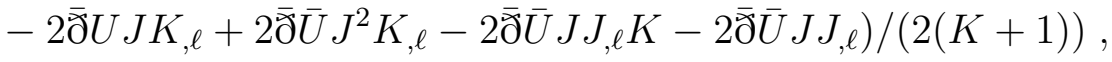

$$
\begin{aligned}
& s_{3}=-\left(J^{2} \bar{J}_{, \ell}+J \bar{J} J_{, \ell}-2 J K K_{, \ell}-2 J K_{, \ell}+2 J_{, \ell} K+2 J_{, \ell}\right) /(K+1), \\
& s_{4}=(ð A ð \omega J \bar{J}+2 ð A ð \omega K+2 ð A ð \omega-ð A \bar{\partial} \omega J K \\
& \left.-ð A \bar{\partial} \omega J-\partial \omega \bar{\partial} A J K-\partial \omega \bar{\partial} A J+\bar{\partial} A \bar{\partial} \omega J^{2}\right) /(2(K+1)), \\
& s_{5}=\left(2 \widetilde{\partial}^{2} A J \bar{J}+4 ð^{2} A K+4 \widetilde{\partial}^{2} A+2 \bar{\partial}^{2} A J^{2}-4 \bar{\varnothing} ð A J K\right. \\
& -4 \bar{\partial} \partial A J+ð A ð J J \bar{J}^{2}+2 ð A ð J \bar{J} K+2 ð A ð J \bar{J}+ð A ð \bar{J} J^{2} \bar{J} \\
& \text { + } 2 \text { ðð } \bar{J} J K+2 ð A ð \bar{J} J-2 ð A ð K J \bar{J} K-4 ð A ð K J \bar{J}-4 ð A ð K K \\
& -4 ð A ð K-\text { ð } A \bar{\partial} J J \bar{J} K+2 ð A \bar{\partial} J K+2 ð A \bar{\partial} J-\partial A \bar{\partial} \bar{J} J^{2} K
\end{aligned}
$$

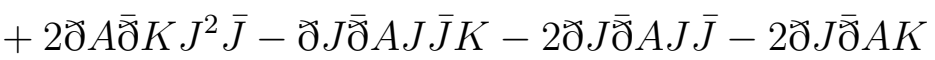

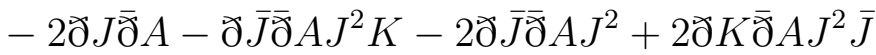

$$
\begin{aligned}
& +4 ð K \bar{\partial} A J K+4 \check{\partial} K \bar{\partial} A J+\bar{\varnothing} A \bar{\partial} J J^{2} \bar{J} \\
& \left.+\bar{\varnothing} A \bar{\varnothing} \bar{J} J^{3}-2 \bar{ð} A \bar{\varnothing} K J^{2} K\right) /(4(K+1)) \text {. }
\end{aligned}
$$

In our implementation,,$l$ derivatives are derived from spectrally calculated ${ }_{,}$ derivatives using the appropriate Jacobian. 


\section{Results}

In our comparison tests of CCE, the worldtube boundary data were extracted from a simulation of an equal mass non-spinning head-on black hole collision, with initial separation of $30 \mathrm{M}$. The control run (Isotropic) utilized the standard harmonic gauge damping identical to that in [23] throughout the head-on merger and ring-down. Harmonic gauge damping adds a dissipative forcing term to the wave equations satisfied by the harmonic Cartesian spatial coordinates $(x, y, z)$. In order to diminish the effects of a custom designed gauge, we also compare with results of another run (HytZero) which turns off gauge damping in the harmonic $y$-direction, transverse to the $x$-direction motion of the black holes. These two high-resolution runs were used as boundary data for all the subsequent CCE runs. These runs include 3 different resolutions, 2 different codes, 2 different gauges, and 3 different extraction radii, for a total of 36 runs.

As described in [15], the SpEC characteristic evolution algorithm exploits spectral methods and innovative integral methods that greatly improve upon the speed and accuracy of the Pitt null code. This is seen as essential for for taking advantage of the efficiency of SpEC Cauchy evolution. The necessary improvement in efficiency has been preserved in the SpEC extraction module, as displayed in Table 1. The comparison runs were performed using the current version of the Pitt code [20], which forms part of the Einstein Toolkit.

The initial conditions and extraction parameters were deliberately chosen as a stressful test of the algorithms. In particular, at the beginning of the run the black hole excision boundary extends out to Cartesian radius $R=16 M$, which is very close to our smallest choice of extraction radius at $R=30 M$. At this radius, gauge effects are highly significant and would make perturbative extraction schemes meaningless, 
in accordance with our intentions. One consequence of such an extreme choice is that differences between the Pitt and SpEC inertial frame and worldtube initialization procedures lead to noticeably different waveforms. Worldtube initialization involves supplying the "integration constants" from the Cauchy code, which allows radial integration of the characteristic hypersurface and evolution equations 2.3 - 2.7 from the worldtube to $\mathscr{I}^{+}$. In both Pitt null code and SpEC, the initial condition on $J$ is determined by the inner boundary value, supplied by the Cauchy evolution, with a smooth roll off to zero at $\mathscr{I}^{+}$.

The extraction worldtube $\Gamma$ is determined by a surface of constant Cartesian radius $R$. In the Pitt CCE code, the areal radius $r_{w t}$ of $\Gamma$ lies between two surfaces of constant Cartesian radii $R_{1} \leq r_{w t} \leq R_{2}$ and this carries over to the compactified radial coordinate. As a result, interpolation is necessary to supply the integration constants, which introduces numerical error. In the SpEC CCE code, this interpolation error is avoided by introducing the compactified radial coordinate $(2.9)$, with range $1 / 2 \leq \rho \leq 1$ between $\Gamma$ and $\mathscr{I}^{+}$.

Worldtube data from each run were extracted using both Pitt and SpEC CCE, at three different Cartesian radii: $R=30 M, R=100 M$ and $R=250 M$, as illustrated by the news function waveforms in Figs. 2, 4, and 6, respectively. In these figures, the HytZero and Isotropic waveforms are so close that they appear on top of one another. The major discrepancy between the Pitt and SpEC waveforms is due to the worldtube interpolation error in the Pitt code. This is especially evident at small extraction radii, where there is strong "junk" radiation near the worldtube, which is inherent in the initial Cauchy data and its mismatch with the initial characteristic data.

This interpolation error in the Pitt code converges away at larger radii, where the field gradients between $R_{1}$ and $R_{2}$ become smaller. This is seen in Figs. 3, 5, and 


\begin{tabular}{|r|ccc|ccc|}
\hline Run & Pitt1 & Pitt2 & Pitt3 & SpEC1 & SpEC2 & SpEC3 \\
\hline$N_{r}$ & 100 & 150 & 200 & 10 & 12 & 14 \\
$N$ or $L$ & 40 & 60 & 80 & 12 & 14 & 17 \\
$\Delta t / M$ & 0.1 & $0.0666 \ldots$ & 0.05 & 1.0 & $0.666 \ldots$ & 0.5 \\
$\mathrm{~T}$ (CPU hours) & 173 & 274 & 374 & 0.7 & 1.9 & 3.1 \\
\hline
\end{tabular}

Table 1: Resolution parameters used for code convergence comparisons, with time steps $\Delta t . N_{r}$ represents the radial grid sizes. The Pitt null code uses two stereographic patches with $2 N^{2}$ total number of angular grid points. The SpEC code has $2 L^{2}$ total angular grid points. $\mathrm{T}$ is the $\mathrm{CPU}$ time taken for $R=30 M, t_{\text {final }}=450 M$ runs in the Isotropic gauge, and is representative for the other runs. All resolutions and codes were run from the same initial data.

7. where the relative difference between the Pitt and SpEC news function waveforms is compared with the relative numerical error implied by convergence tests.

Each run was computed at 3 different resolutions to monitor convergence, as indicated in Table 1. In the following subsections, we first show convergence and the removal of gauge effects, separately for the Pitt and SpEC codes. Next, we compare $\Psi_{4}^{0}$ waveforms and establish further agreement between the two codes. Finally, we

examine the evolution of the inertial coordinates at $\mathscr{I}^{+}$relative to the worldtube coordinates induced by the Cauchy evolution.

Comparison of the relative error $E_{r e l}$ between dataset $A$ and dataset $B$ is computed according to

$$
E_{r e l}=\log _{10}\left(\frac{|A-B|}{|B|}\right)
$$

where in convergence tests $B$ is the highest resolution dataset, and the real parts of the $(\ell, m)=(2,2)$ spherical harmonic modes are compared. 


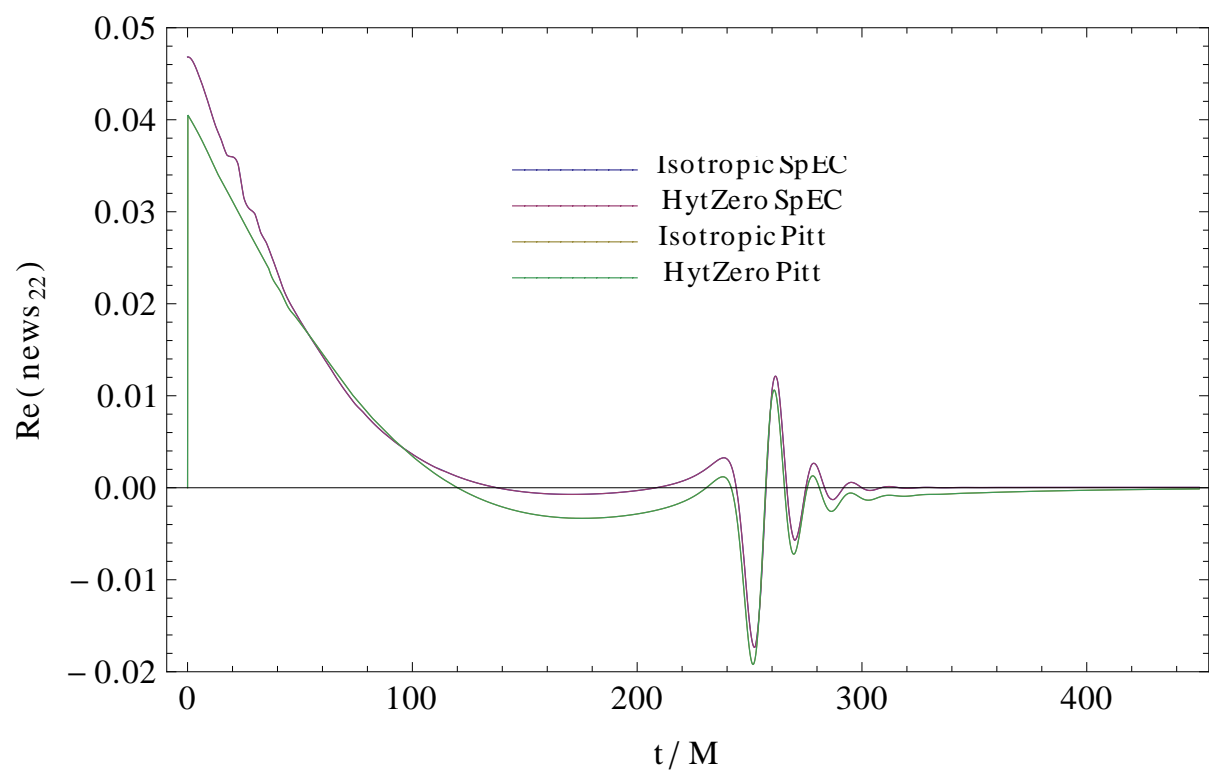

Figure 2: Waveforms of the real part of the $(2,2)$ spherical harmonic mode of the news function, as computed by the Pitt null code and SpEC with extraction worldtube at $R=30 M$. Different initialization procedures at the worldtube give rise to a difference between the Pitt and SpEC waveforms, which is most pronounced at this small extraction radius. The different gauge choices, Isotropic and HytZero, do not have noticeable effect on this scale, indicating successful gauge effect removal in both codes.

\subsection{Pitt code convergence and removal of gauge effects}

Here, in order to establish a baseline, we examine the self convergence of the Pitt code for each of the extraction radii, using the three resolutions (Pitt1,Pitt2,Pitt3) indicated in Table 1. In Figs. 8, 9, and 10, we see in both the Isotropic and HytZero gauges that the news function converges over the entire run. Indeed, Isotropic (solid lines) and HytZero (dashed lines) overlap completely. The figures also plot the relative error in the news computed in both gauges, which is consistently below the numerical error implied by convergence tests for extraction worldtubes at $R=30 M$ and $R=100 M$. This verifies that the Pitt code successfully removes gauge effects. Furthermore, the figures plot the relative error between the news computed in the 


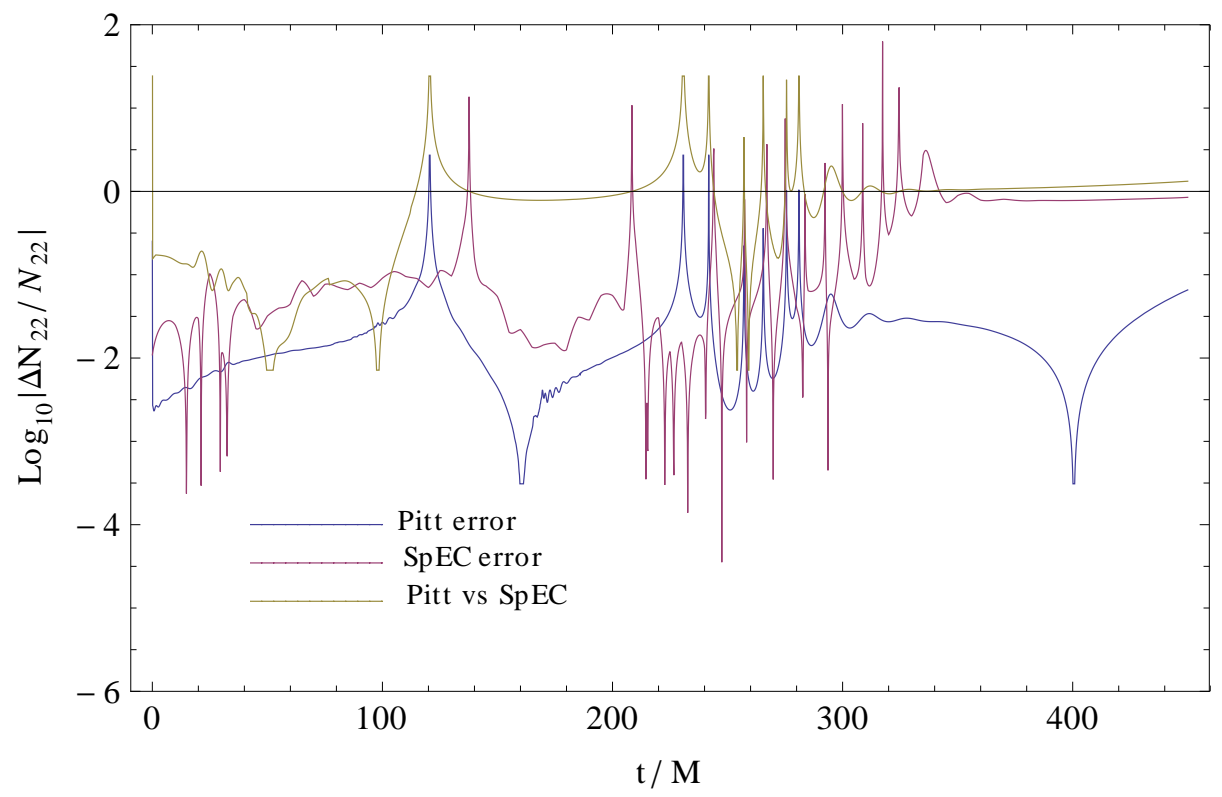

Figure 3: Graphs showing the relative difference between the real part of the $(2,2)$ mode Pitt and SpEC news function waveforms for extraction radius $R=30 M$, in comparison to the relative numerical error implied by convergence tests, corresponding to the waveforms in Fig. 2, While both SpEC and Pitt have comparable and consistent levels of error, the codes do not agree within that level of error at this extraction radius.

worldtube coordinates and the inertial coordinates at $\mathscr{I}^{+}$. In the $R=30 M$ case shown in Fig. 8, the initial discrepancy is high due to the strong gauge effects of junk radiation. It does not fall below the relative error between the Isotropic and HytZero gauges until well after the signal has passed. This confirms that the transformation to inertial coordinates is essential for correctly removing gauge effects from the waveform. For extraction at $R=100 M$ shown in Fig. 9, the relative error between worldtube and inertial coordinates has dropped below the Isotropic-HytZero gauge effect. At $R=250 M$ shown in Fig. 10 , the predominant error is the Isotropic-HytZero gauge effect.

These results show that the selected runs do produce a substantial gauge error between the worldtube and inertial coordinates and that the Pitt code effectively 


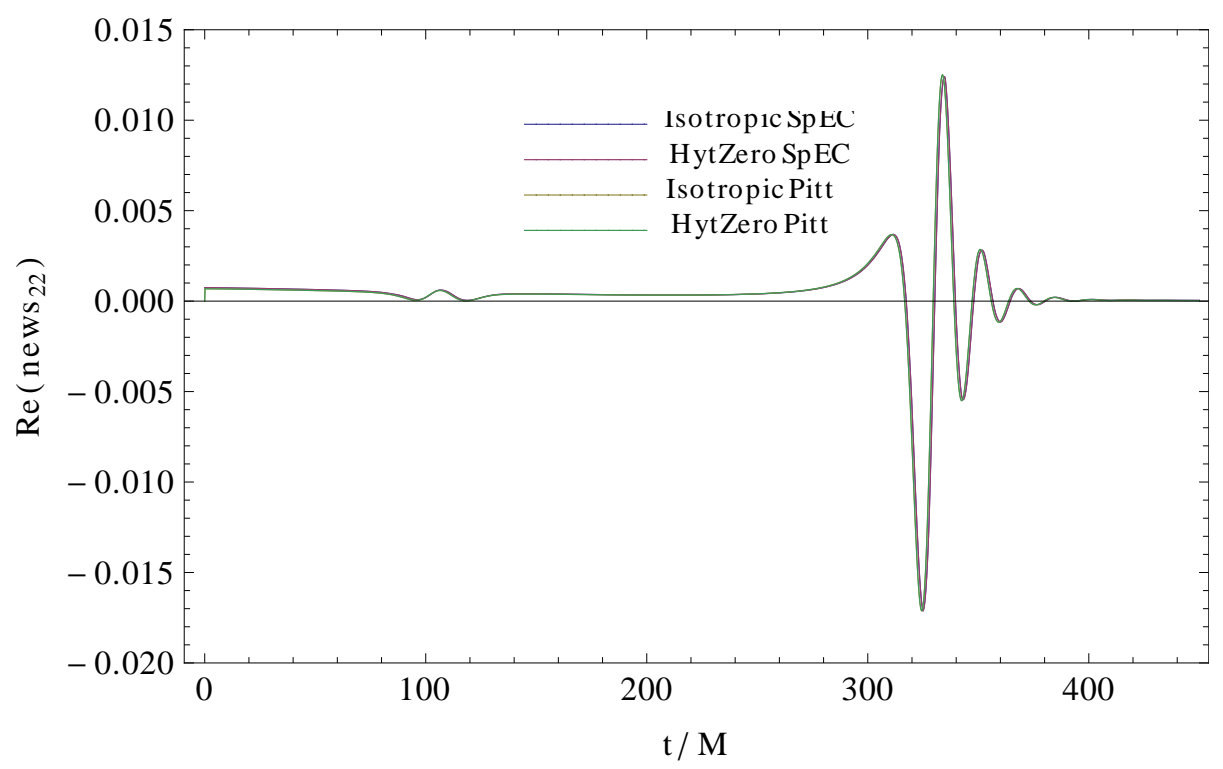

Figure 4: Waveforms of the real part of the $(2,2)$ mode of the news function, as computed by the Pitt and SpEC codes with extraction worldtube at $R=100 M$. Compared to Fig. 2, at this larger extraction radius the worldtube initialization differences lead to a much smaller difference between waveforms, which appear nearly identical here. The main discrepancy arises from the treatment of the junk radiation at early times. Here, too, gauge differences between HytZero and Isotropic are not visible at this scale.

removes it, while remaining convergent for the duration of the run.

\subsection{SpEC code convergence and removal of gauge effects}

Here we examine the SpEC code's self convergence for each extraction radii, in the same way that the Pitt code was examined in Sec. 5.1. In Figs. 11, 12, and 13, we see that convergence, measured with the resolutions indicated in Table 1, is comparable to the Pitt code's convergence, while the potential gauge contamination is consistently removed at all worldtube radii. As in Figs. 8, 9, and 10, the solid lines (Isotropic) and dashed lines (HytZero) overlap due to consistency in gauge removal. The SpEC extraction code effectively removes gauge error at all radii while remaining convergent 


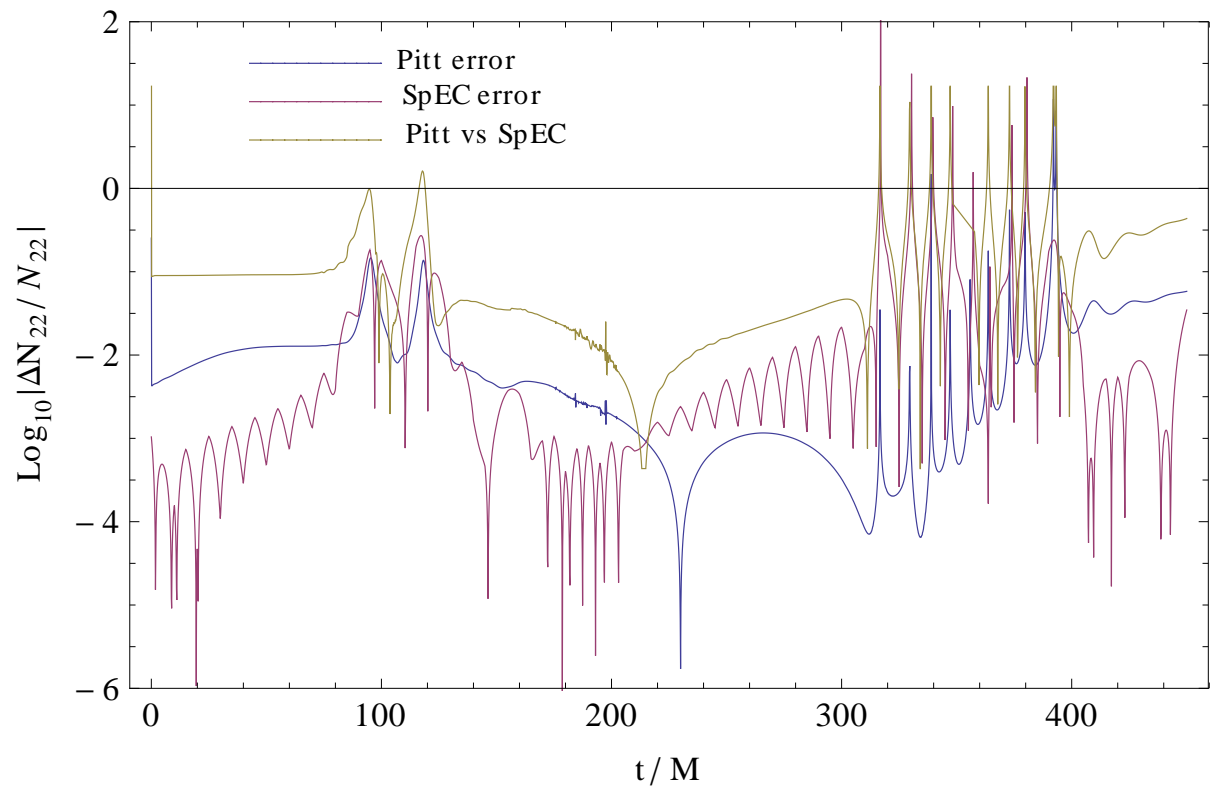

Figure 5: Graph showing the relative difference between the real part of the $(2,2)$ mode SpEC and Pitt news waveforms for extraction radius $R=100 M$, and the relative numerical error, corresponding to the waveforms in Fig. 4. In comparison with Fig. 3 , by $R=100 M$ the difference between the Pitt and SpEC algorithms has dropped to the level of numerical error in each algorithm.

throughout the runs.

\subsection{Comparison of $\Psi_{4}^{0}$ between the Pitt and SpEC codes}

In Secs 5.1 and 5.2 , we have shown that both codes are convergent and remove potential gauge effects. We have also demonstrated that the difference in the news computed by the two codes disappears as the extraction worldtube radius increases. Here we provide further evidence that even at a small worldtube radius the waveform computed by the SpEC code is valid.

After the gauge freedom is removed by extraction, there is still supertranslation and Lorentz freedom in the choice of inertial coordinates, which affect the phase and velocity of the inertial observers. This effect is highly sensitive to initial conditions 


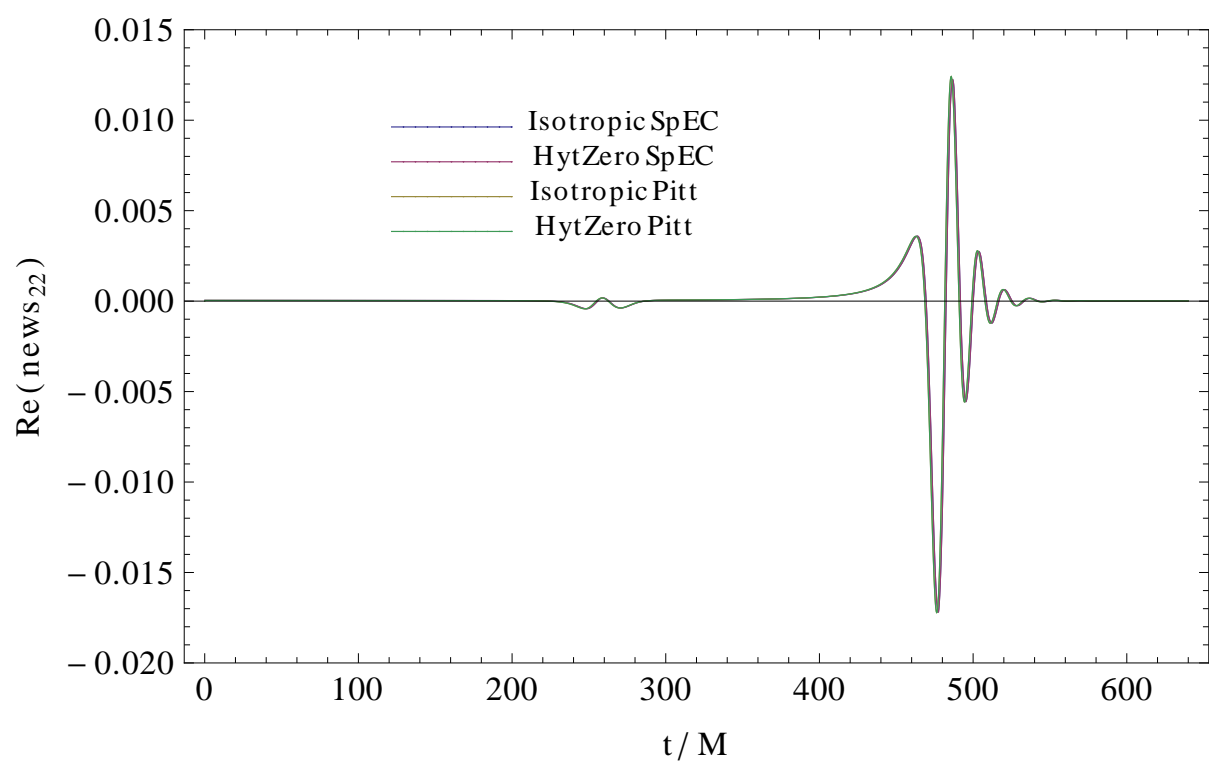

Figure 6: Waveforms of the real part of the $(2,2)$ mode of the news function, as computed by the Pitt and SpEC codes with extraction worldtube at $R=250 M$. At this large extraction radius there is a barely noticeable difference between all the waveforms, limited to the junk radiation at early times.

and also to the evolution of the inertial confomal transformation factor $\omega$, especially in the extreme gauge conditions of extraction at $R=30 M$. It feeds into the worldtube interpolation error in the Pitt code. In order to verify that the discrepancy illustrated in Fig. 2 between the news computed by the Pitt null code and SpEC is partially due to this inertial coordinate freedom, we compute the time derivative of the news, which is related to the Weyl curvature in inertial coordinates according to $\partial_{t} N=\Psi_{4}^{0}$. This suppresses phase differences between the two waveforms. In making the comparisons, $\Psi_{4}^{0}$ is computed semi-independently using the Weyl tensor waveform module in the current version of the Pitt code [20]. In these runs, $\Psi_{4}^{0}$ was found to be convergent with truncation error comparable to the consistency between $\Psi_{4}^{0}$ and $\partial_{t} N$ in the Pitt code.

In Fig. 14, we see that the time derivative of the news and $\Psi_{4}^{0}$ have much less 


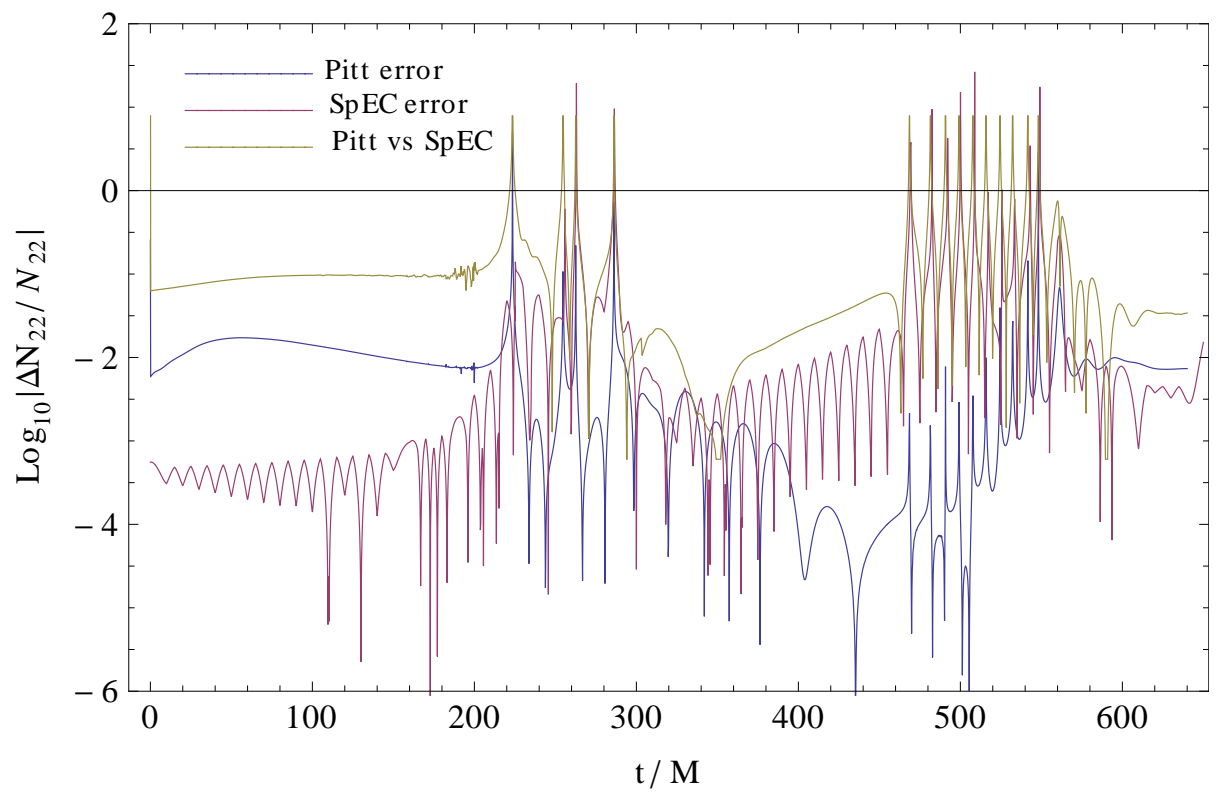

Figure 7: Graphs showing the relative difference between SpEC and Pitt news waveforms at $R=250 M$, in comparison to the relative numerical error, corresponding to the waveforms in Fig. 6. During the post-junk part of the waveform, the error due to worldtube initialization has dropped to the level of the numerical error, completing the trend seen in Fig. 5 .

discrepancy than Fig. 2 would suggest. In Figs. 15, 17, and 19, we compare relative errors between $\Psi_{4}^{0}$ and $\partial_{t} N$ computed by the Pitt and SpEC codes. Not only is there agreement between the codes at $R=30 M$, this agreement persists for larger extraction radii, as shown in Figs. 16 and 18. Both codes show agreement with the $\Psi_{4}^{0}$ waveform throughout the runs at all three extraction radii. This indicates that a major part of the discrepancy in Fig. 2 is due to initialization errors in the Pitt code, confirming the physical validity of the extracted SpEC waveform.

\subsection{Relative motion between inertial and worldtube coordinates}

In Section 3, we discussed the construction of an inertial coordinate system and its evolution with respect to the worldtube coordinates constructed from the Cartesian coordinates of the Cauchy code. Here, we describe the motion of the inertial $(\theta, \phi)$ 


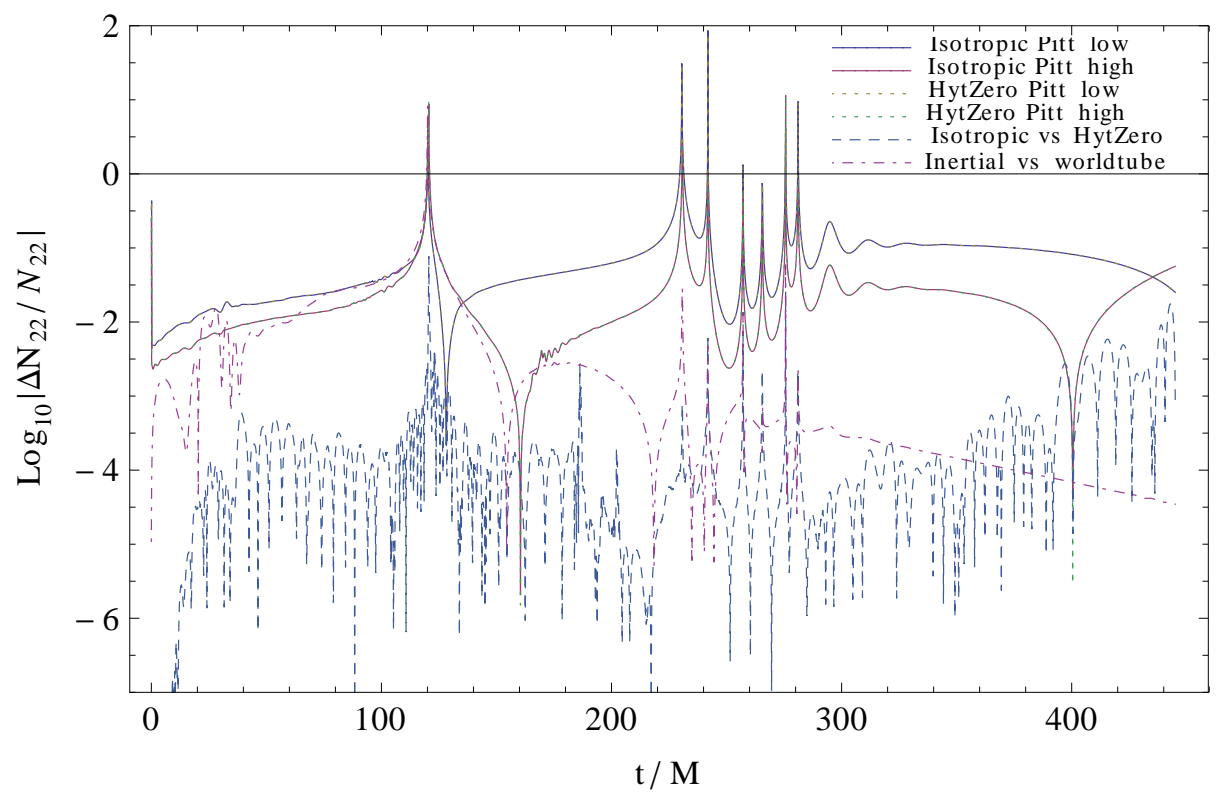

Figure 8: Graphs of the relative error $\log _{10}\left|\Delta N_{22} / N_{22}\right|$ in the $(2,2)$ mode of the news function for the $R=30 M$ Pitt extraction run in both gauges. The relative errors for the Pitt1 (low) and Pitt2 (high) resolutions (compared to Pitt2 and Pitt3 respectively) are rescaled to demonstrate convergence. The dashed blue line indicates the relative error (Isotropic vs HytZero) between the news computed in both gauges. The dot-dashed purple line (Inertial vs worldtube) indicates the relative error between the news computed in the worldtube coordinates and the inertial coordinates. At this small extraction radius, this discrepancy is high due to the strong gauge effect of junk radiation.

angular coordinates relative to the worldtube angular coordinates, constructed in the standard way from the worldtube Cartesian coordinates. Figure 20 illustrates the global pattern of this relative motion for the Isotropic gauge SpEC run with the highest resolution extraction at $R=30 M$. Generally speaking, the coordinates wiggle back and forth in the direction corresponding to the motion of the black holes. The complete movement amounts to at most a few percent of their initial values, but even this is sufficient to introduce considerable gauge error in the waveform, as already seen in Fig. 8. 


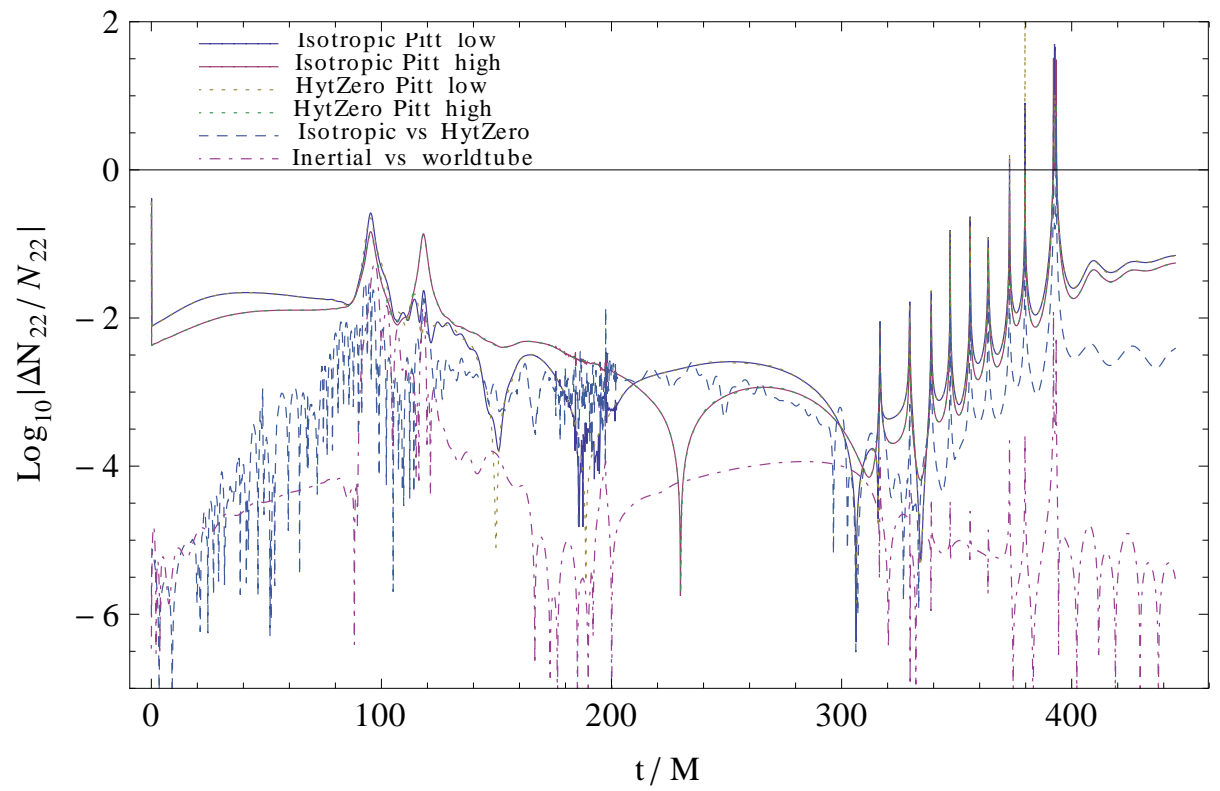

Figure 9: Graphs of the relative error $\log _{10}\left|\Delta N_{22} / N_{22}\right|$ in the news function for Pitt extraction at $R=100 M$. Again, the errors for the Pitt1 and Pitt2 resolutions demonstrate convergence. Compared to Fig. 8, the relative error (Inertial vs worldtube) between inertial and worldtube coordinates has now dropped below the Isotropic vs HytZero gauge effect.

In Fig. 21, the relative $\phi$ motion of the point circled in Fig. 20 is plotted as a function of inertial time. Initial junk radiation causes considerable wobble, followed by a smooth return almost to its starting point. The maximum excursion of the $\phi$-coordinate shift from its initial value is about $3.5 \%$.

\subsection{Precessing, spinning binary black hole merger}

In addition to the head-on collision tests which we have described, we have also investigated stability and convergence of the Pitt and SpEC CCE modules, together with the inertial-worldtube coordinate transformation, using the generic test run of precessing, spinning binary black holes in [15], as taken from Taylor et al. [24]. Its parameters are mass ratio $q=3$, black hole spins $\chi_{1}=(0.7,0,0.7) / \sqrt{2}$ and $\chi_{2}=$ $(-0.3,0,0.3) / \sqrt{2}$, number of orbits 26 , total time $T=7509 M$, initial eccentricity 


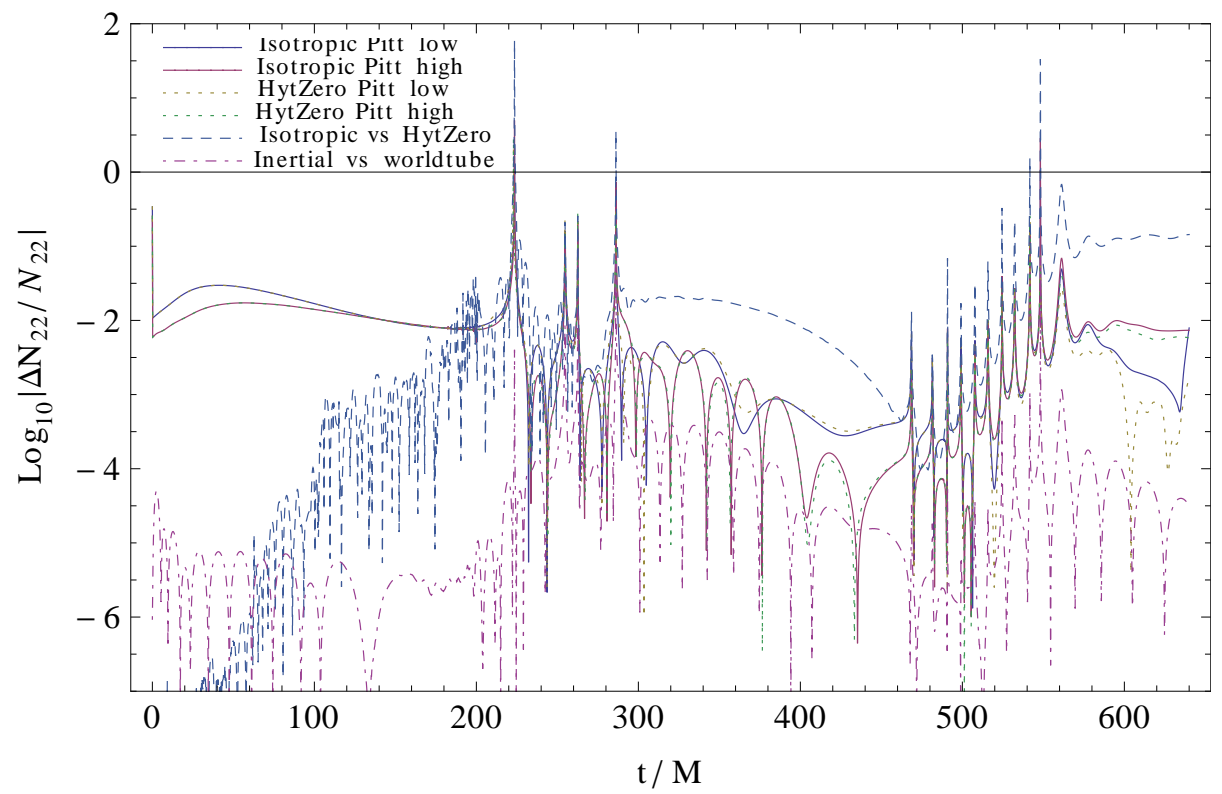

Figure 10: Graphs of the relative error $\log _{10}\left|\Delta N_{22} / N_{22}\right|$ in the news function for Pitt extraction at $R=250 M$. At this large extraction radius, the dominant error arises from the Isotropic-HytZero gauge effect.

$10^{-3}$, initial frequency $\omega_{i n i}=0.032 / M$, and extraction radius $R=100 M$. The Pitt and SpEC waveforms displayed in Fig. 22 are fairly typical waveforms, spanning the initial junk radiation through inspiral, merger, and ringdown.

Figures 23 and 24 show a log scale comparison of the waveforms with absolute error. The codes agree strongly throughout the run.

The relative inertial-worldtube coordinate motion of a representative point in the extended generic run is illustrated in Fig. 25. At early times, the helix has two loops per cycle corresponding to each of the black holes. At later times, precession dominates the evolution of this particular coordinate. Throughout the run, the deviation is around $0.5 \%$. 


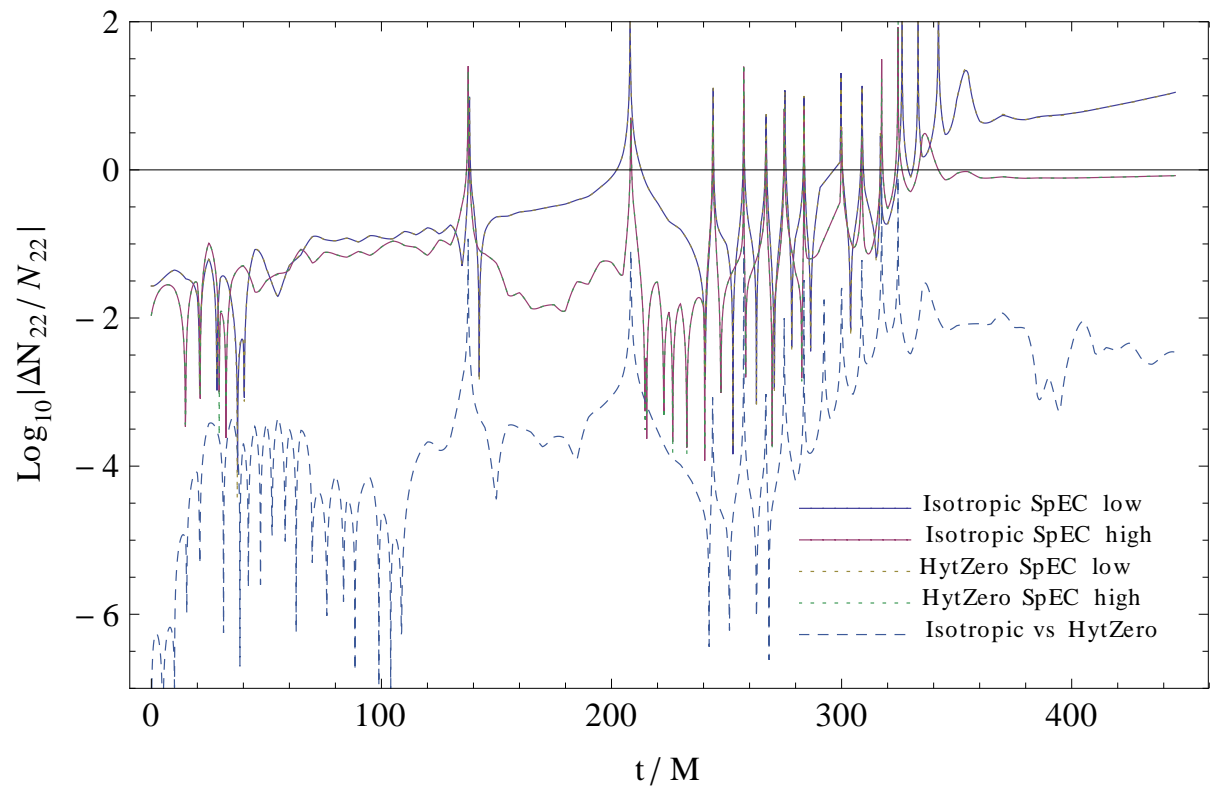

Figure 11: Graphs of the relative error $\log _{10}\left|\Delta N_{22} / N_{22}\right|$ in the news function for the $R=30 M$ SpEC extraction run in both gauges. The relative errors for the SpEC1 (low) and SpEC2 (high) resolutions (compared to SpEC2 and SpEC3 respectively) are rescaled to demonstrate convergence. The graph Isotropic vs HytZero indicates the relative error between the news computed in both gauges. Even at this small extraction radius, there is relatively little Isotropic vs HytZero gauge error.

\section{Conclusion}

The SpEC characteristic evolution algorithm has now been furnished with a convergent, efficient news extraction module. SpEC is now capable of rapidly producing accurate, gauge free waveforms as required.

\section{Acknowledgments}

We thank Nicholas Taylor for his generic spinning binary black hole run that we used to test and baseline code performance. We thank Yosef Zlochower for supplying details of the news module in the Pitt null code. We thank Mark Scheel, Yanbei 


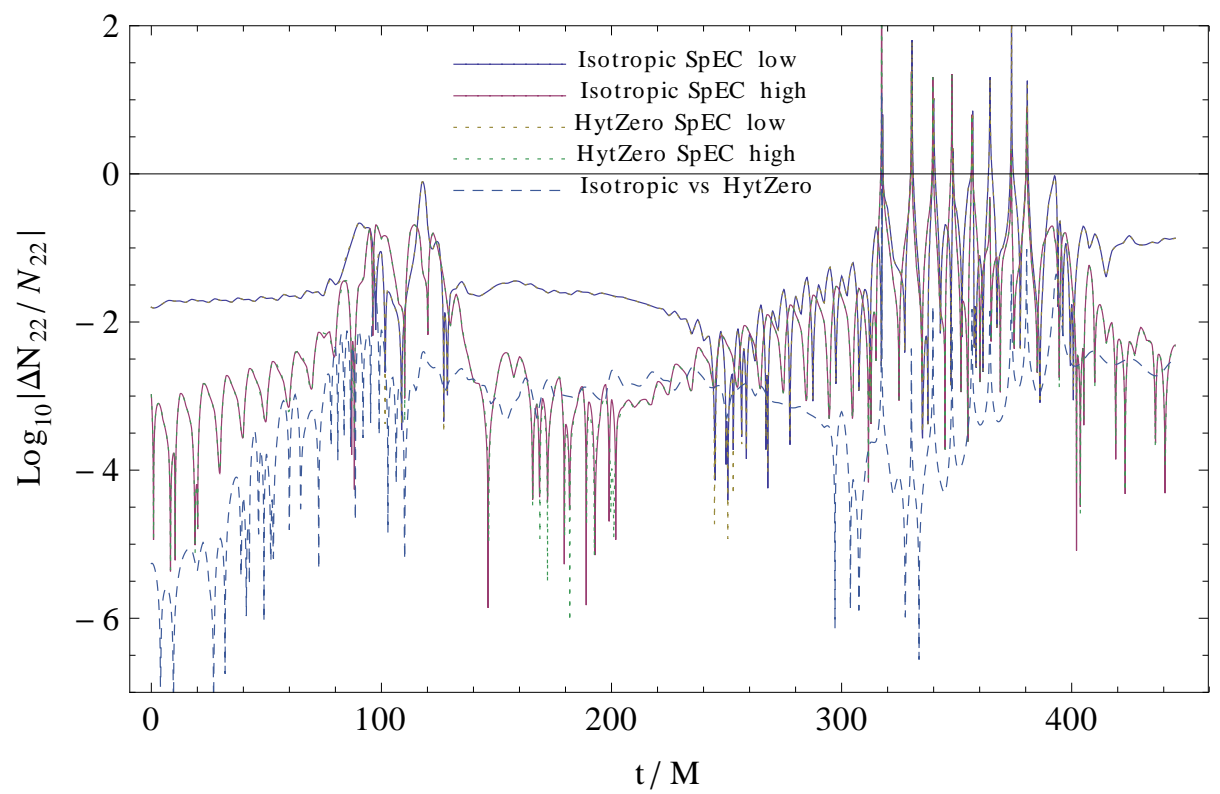

Figure 12: Graphs of the relative error $\log _{10}\left|\Delta N_{22} / N_{22}\right|$ in the news function for the $R=100 M$ SpEC extraction run in both gauges. The graphs show convergence in both gauges. The Isotropic vs HytZero gauge error is relatively small.

Chen, and Christian Reisswig for their advice, support, and technical expertise, and thank Michael Boyle for comments on the manuscript. This research used the Spectral Einstein Code (SpEC)[25]. The Caltech cluster zwicky.cacr.caltech.edu is an essential resource for $\mathrm{SpEC}$ related research, supported by the Sherman Fairchild Foundation and by NSF award PHY-0960291. This research also used the Extreme Science and Engineering Discovery Environment (XSEDE) under grant TG-PHY990002. The UCSD cluster ccom-boom.ucsd.edu was used during code development. This project was supported by the Sherman Fairchild Foundation, and by NSF Grants PHY-1068881, AST-1333520, and CAREER Grant PHY-0956189 at Caltech. JW's research was supported by NSF grant PHY-1201276 to the University of Pittsburgh. 


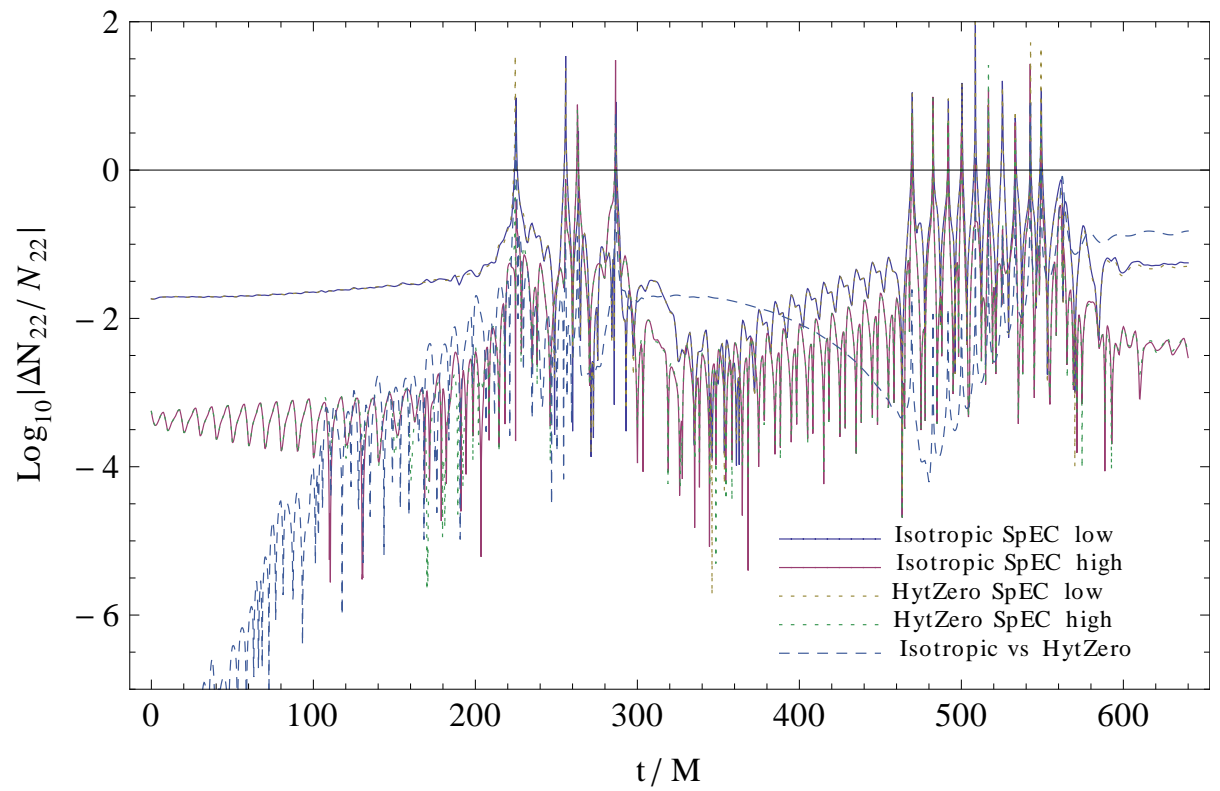

Figure 13: Graphs of the relative error $\log _{10}\left|\Delta N_{22} / N_{22}\right|$ in the news function for the $R=250 M$ SpEC extraction run in both gauges, showing convergence in both gauges as well as small gauge error throughout the run.

\section{References}

[1] S. J. Waldman, "The advanced ligo gravitational wave detector," Tech. Rep.

LIGO-P0900115-v2, LIGO Project, 2011.

[2] T. Accadia, F. Acernese, F. Antonucci, P. Astone, G. Ballardin, et al., "Plans for the upgrade of the gravitational wave detector VIRGO: Advanced VIRGO," in Proceedings of the

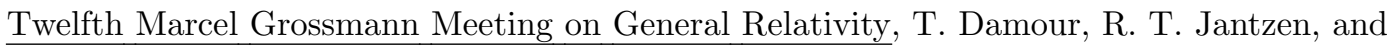
R. Ruffini, eds., pp. 1738-1742. 2009.

[3] LIGO Scientific Collaboration Collaboration, H. Grote, "The GEO 600 status," Class. Quantum Grav. 27 (2010) 084003

[4] K. Somiya and the KAGRA Collaboration, "Detector configuration of KAGRA-the japanese cryogenic gravitational-wave detector," Class. Quantum Grav. 29 no. 12, (2012) 124007.

[5] H. Bondi, M. G. J. van der Burg, and A. W. K. Metzner, "Gravitational waves in general relativity VII. Waves from axi-symmetric isolated systems," Proc. R. Soc. Lond. A 269 (1962) 21-52.

[6] R. K. Sachs, "Gravitational waves in general relativity. VIII. waves in asymptotically flat 


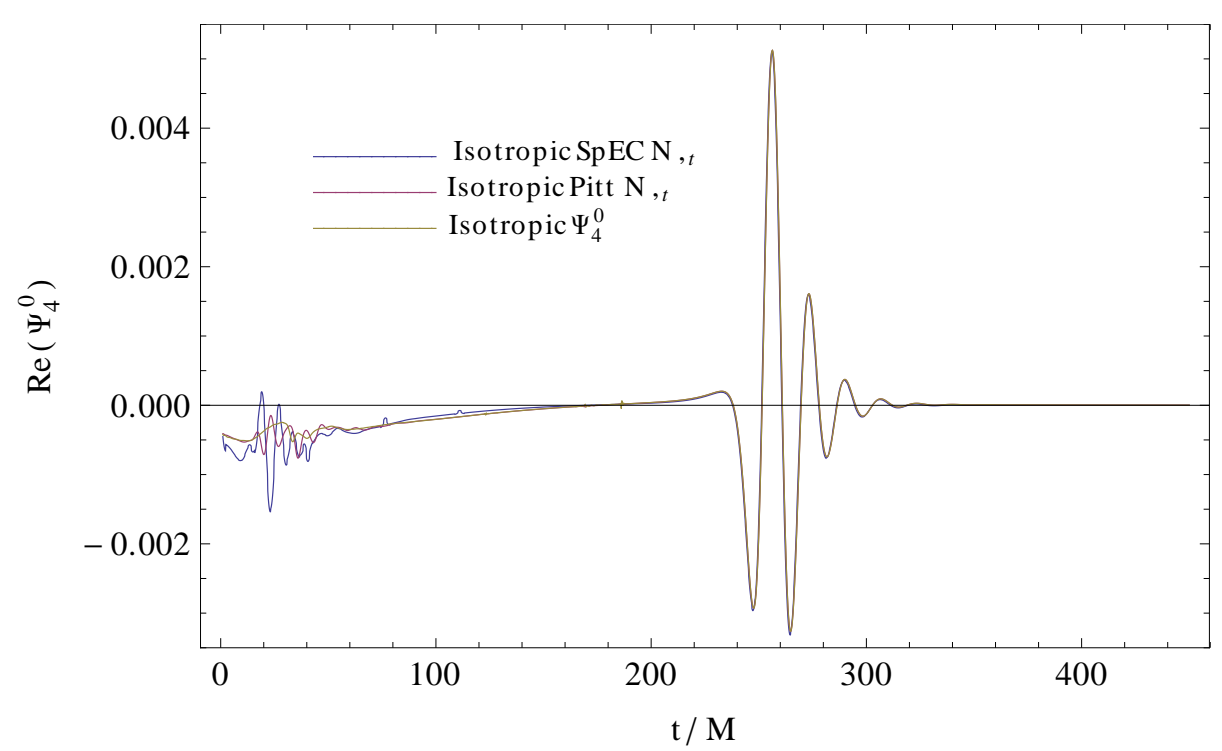

Figure 14: The real part of the $(2,2)$ modes of the waveforms of $\Psi_{4}^{0}$ (computed during the Pitt run) and the time derivative of the news $\partial_{t} N$, as computed for the SpEC and Pitt runs using the Isotropic gauge with extraction radius $R=30 M$. The waveforms are in much better agreement than the comparison of the news waveforms for this extraction radius in Fig. 2.

space-time," Proc. R. Soc. Lond. A 270 no. 1340, (October, 1962) 103-126. http://www.jstor.org/stable/2416200

[7] R. Penrose, "Asymptotic properties of fields and space-times," Phys. Rev. Lett. 10 no. 2, (1963) 66-68.

[8] J. M. Bardeen, O. Sarbach, and L. T. Buchman, "Tetrad formalism for numerical relativity on conformally compactified constant mean curvature hypersurfaces," Phys. Rev. D 83 (2011) 104045.

[9] A. Zenginoğlu, "Hyperboloidal evolution with the Einstein equations," Class. Quantum Grav. 25 (2008) 195025, arXiv:gr-qc/0808.0810.

[10] V. Moncrief and O. Rinne, "Regularity of the Einstein equations at future null infinity," Class. Quantum Grav. 26 (2009) 125010, http://www.arxiv.org/abs/gr-qc/0811.4109

[11] L. A. Tamburino and J. H. Winicour, "Gravitational fields in finite and conformal Bondi frames," Phys. Rev. 150 (1966) 1039-1053. http://link.aps.org/doi/10.1103/PhysRev.150.1039. 


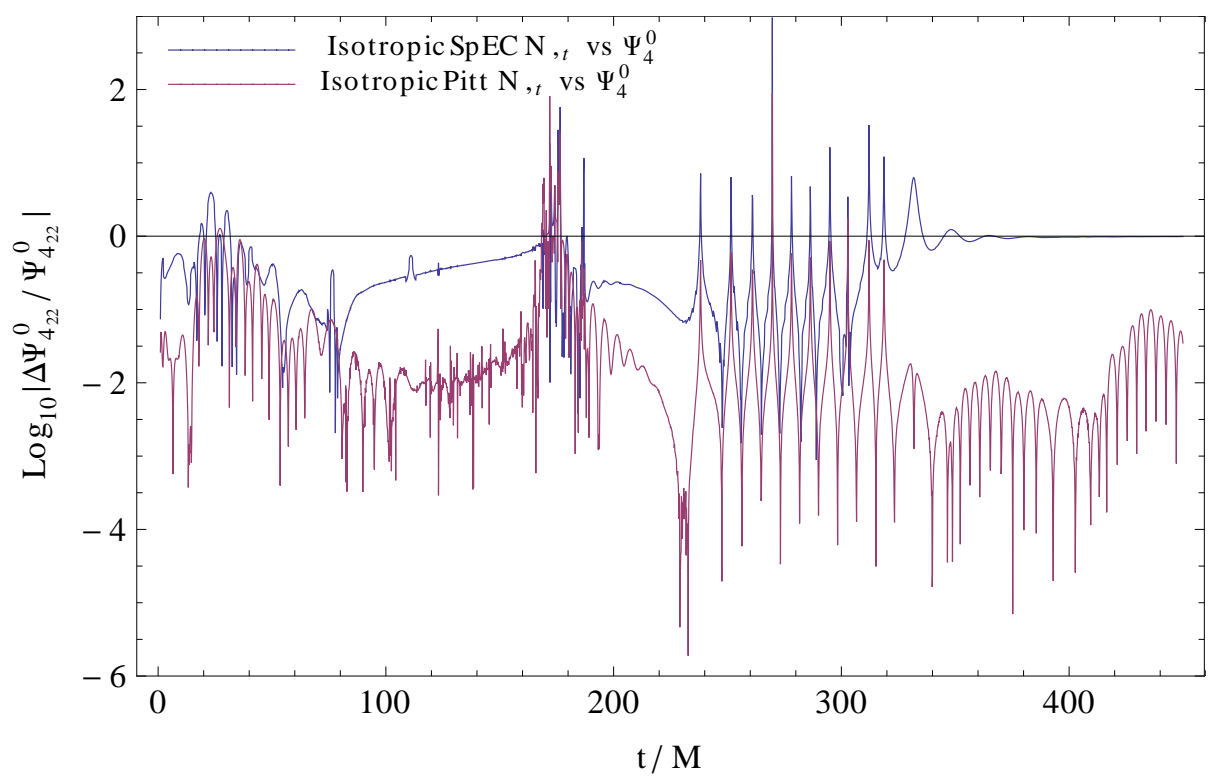

Figure 15: The relative error $\log _{10}\left|\Delta \Psi_{4_{22}}^{0} / \Psi_{4_{22}}^{0}\right|$ between $\Psi_{4}^{0}$ and $\partial_{t} N$ as computed for the SpEC and Pitt runs using the Isotropic gauge with extraction radius $R=30 M$, corresponding to the waveforms in Fig. $14 . \Psi_{4}^{0}$ and its numerical truncation error were computed using the Pitt code. Truncation error in $\Psi_{4}^{0}$ was consistent with the $\partial_{t} N$ error shown here. The good agreement between the SpEC and Pitt results eventually lapses, but not until well after ring-down.

[12] R. A. Isaacson, J. S. Welling, and J. Winicour, "Null cone computation of gravitational radiation," J. Math. Phys. 24 no. 1824, (1983) . http://iopscience.iop.org/0264-9381/30/7/075017.

[13] N. T. Bishop, R. Gomez, L. Lehner, M. Maharaj, and J. Winicour, "High-powered gravitational news," Phys. Rev. D56 (1997) 6298-6309, arXiv:gr-qc/9708065

[14] C. Reisswig, N. T. Bishop, and D. Pollney, "General relativistic null-cone evolutions with a high-order scheme," Gen. Rel. Grav. 45 (5) (2013) 1069-1094, arXiv:1208.3891 [gr-qc].

[15] C. J. Handmer and B. Szilágyi, "Spectral characteristic evolution: A new algorithm for gravitational wave propagation," Classical and Quantum Gravity 32 (2015) 025008, arXiv:1406.7029.

[16] R. Gómez, L. Lehner, P. Papadopoulos, and J. Winicour, "The eth formalism in numerical relativity," Class. Quantum Grav. $(01,1997)$, arXiv:gr-qc/9702002. 


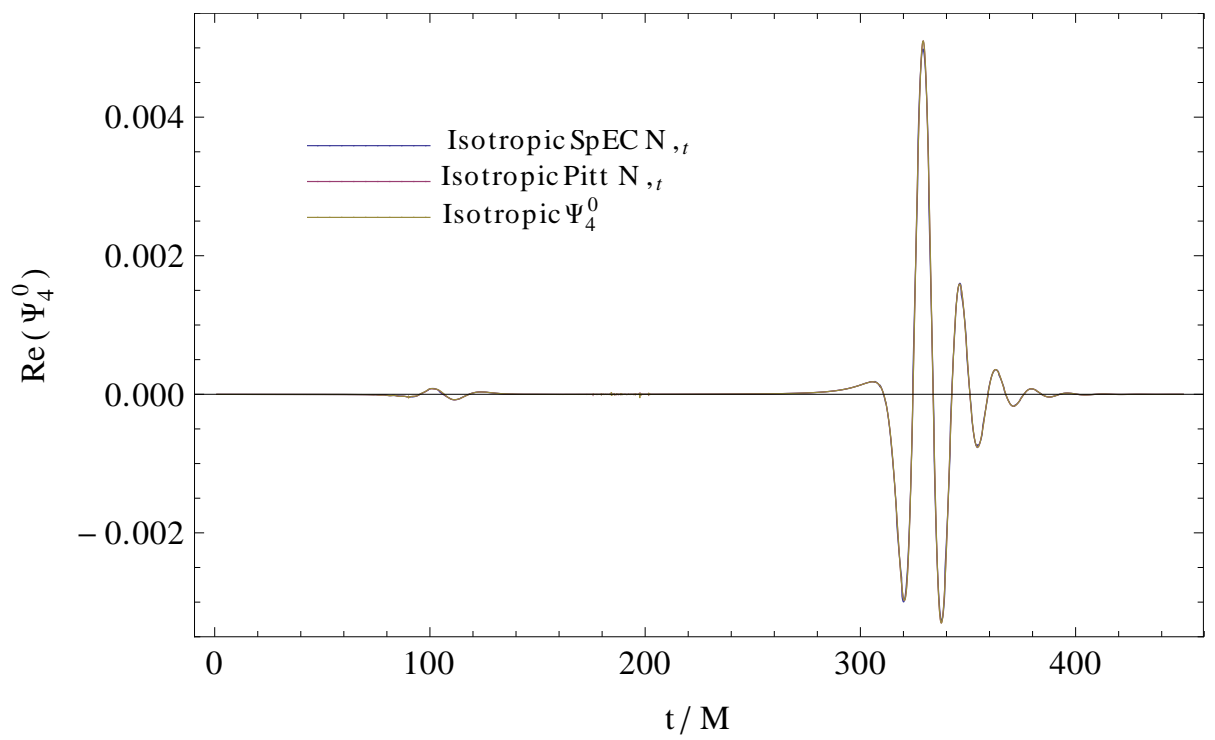

Figure 16: Waveforms of $\Psi_{4}^{0}$ and $\partial_{t} N$ as computed for the SpEC and Pitt runs using the Isotropic gauge with extraction radius $R=100 M$.

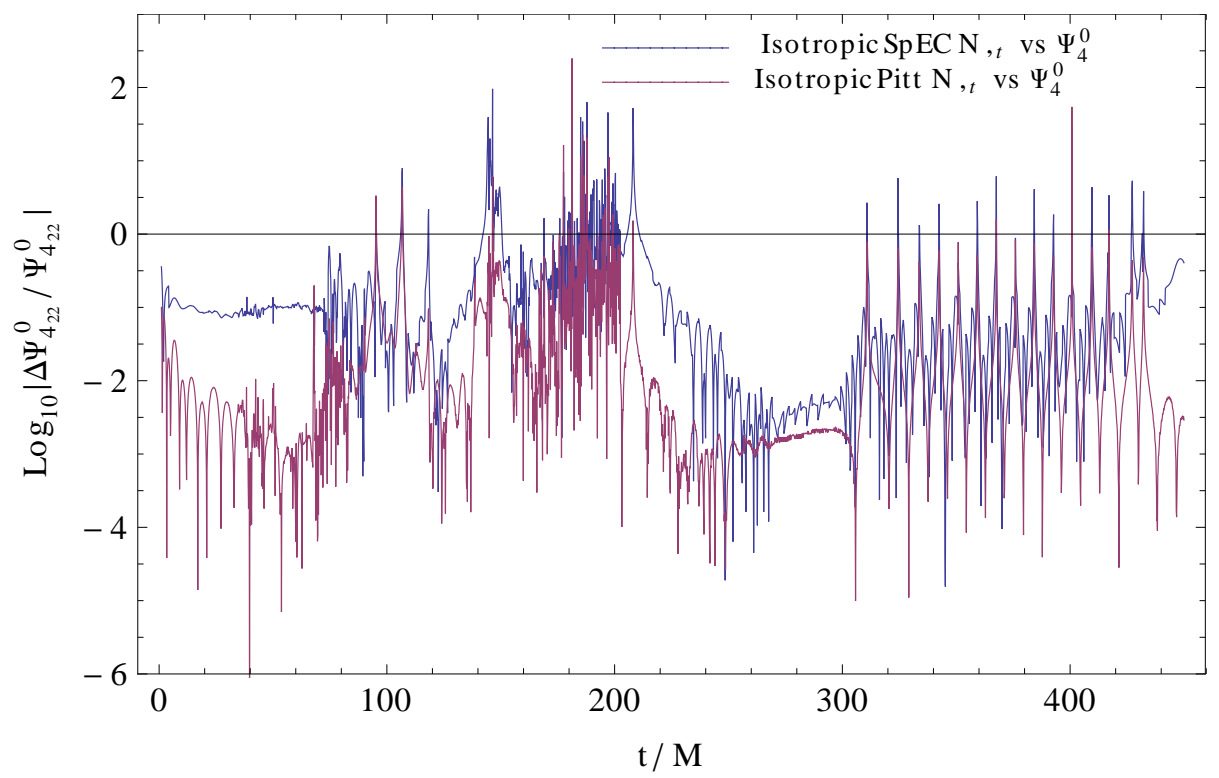

Figure 17: The relative error $\log _{10}\left|\Delta \Psi_{4_{22}}^{0} / \Psi_{4_{22}}^{0}\right|$ between $\Psi_{4}^{0}$ and $\partial_{t} N$ as computed for the SpEC and Pitt runs using the Isotropic gauge with extraction radius $R=100 M$, corresponding to the waveforms in Fig. 16. Both codes show comparable agreement throughout the run. 


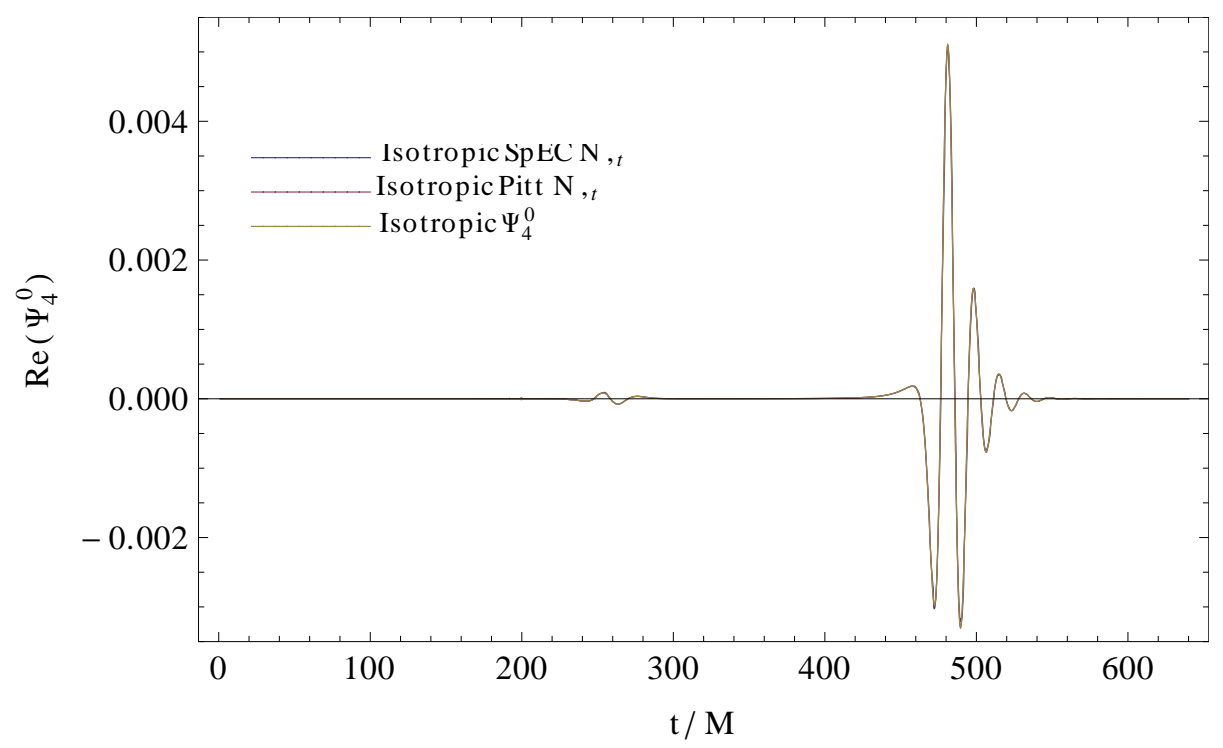

Figure 18: Waveforms of $\Psi_{4}^{0}$ and $\partial_{t} N$ as computed for the SpEC and Pitt codes using the Isotropic gauge with extraction radius $R=250 M$.

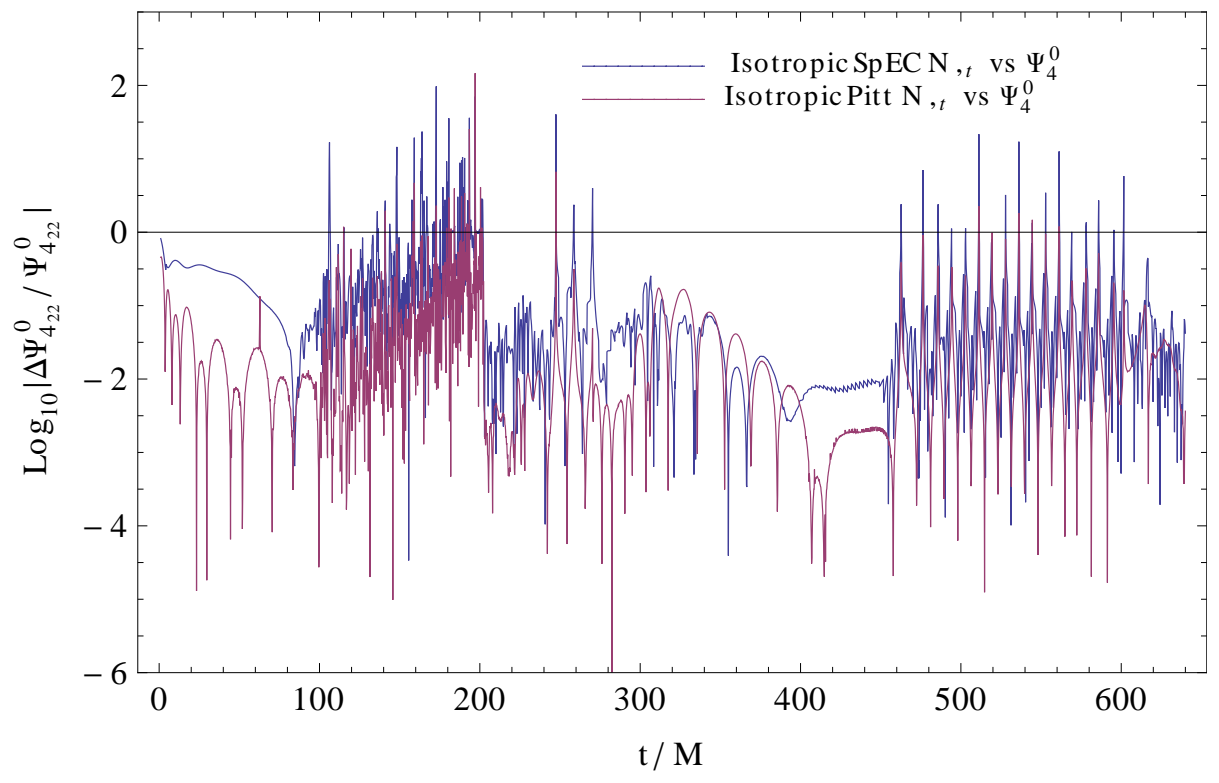

Figure 19: The relative error $\log _{10}\left|\Delta \Psi_{4_{22}}^{0} / \Psi_{4_{22}}^{0}\right|$ between $\Psi_{4}^{0}$ and $\partial_{t} N$ computed for the SpEC and Pitt runs using the Isotropic gauge with extraction radius $R=250 M$, corresponding to the waveforms in Fig. 18. Both codes show good agreement throughout the run. 


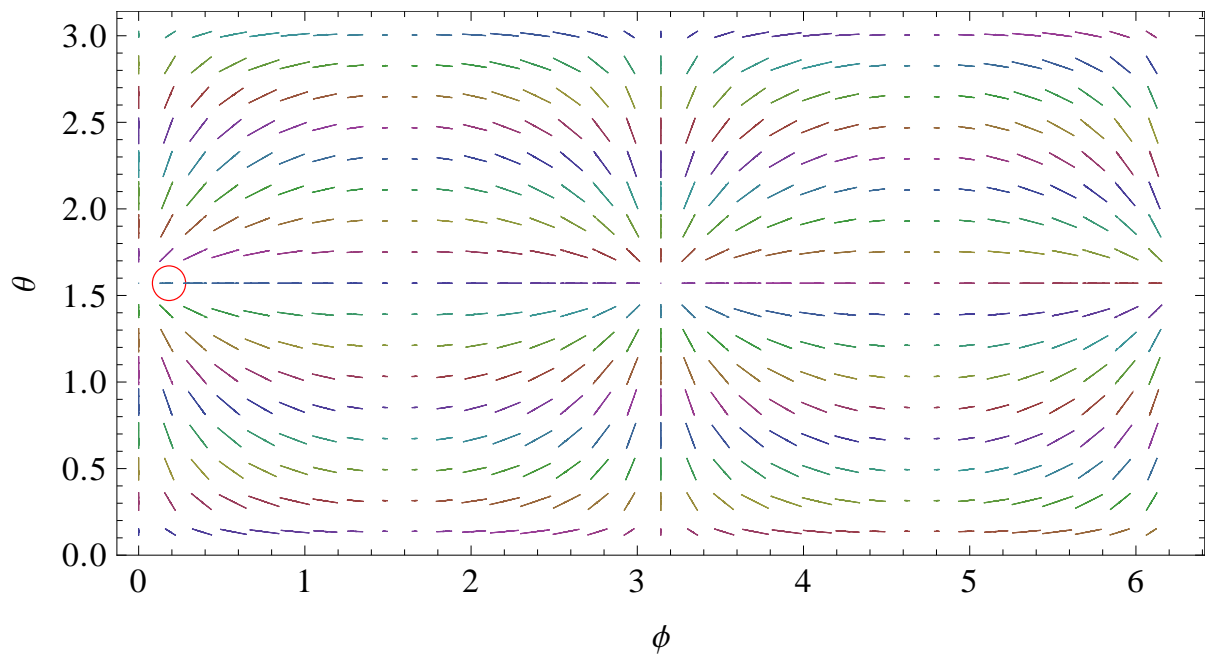

Figure 20: The arrows indicate the global pattern of the motion of the $(\theta, \phi)$ inertial coordinates at each spherical collocation point (labeled in radians) relative to the worldtube angular coordinates. Global motion is primarily in the Cartesian $x$ direction, and has been exaggerated by a factor of 10 for visibility.

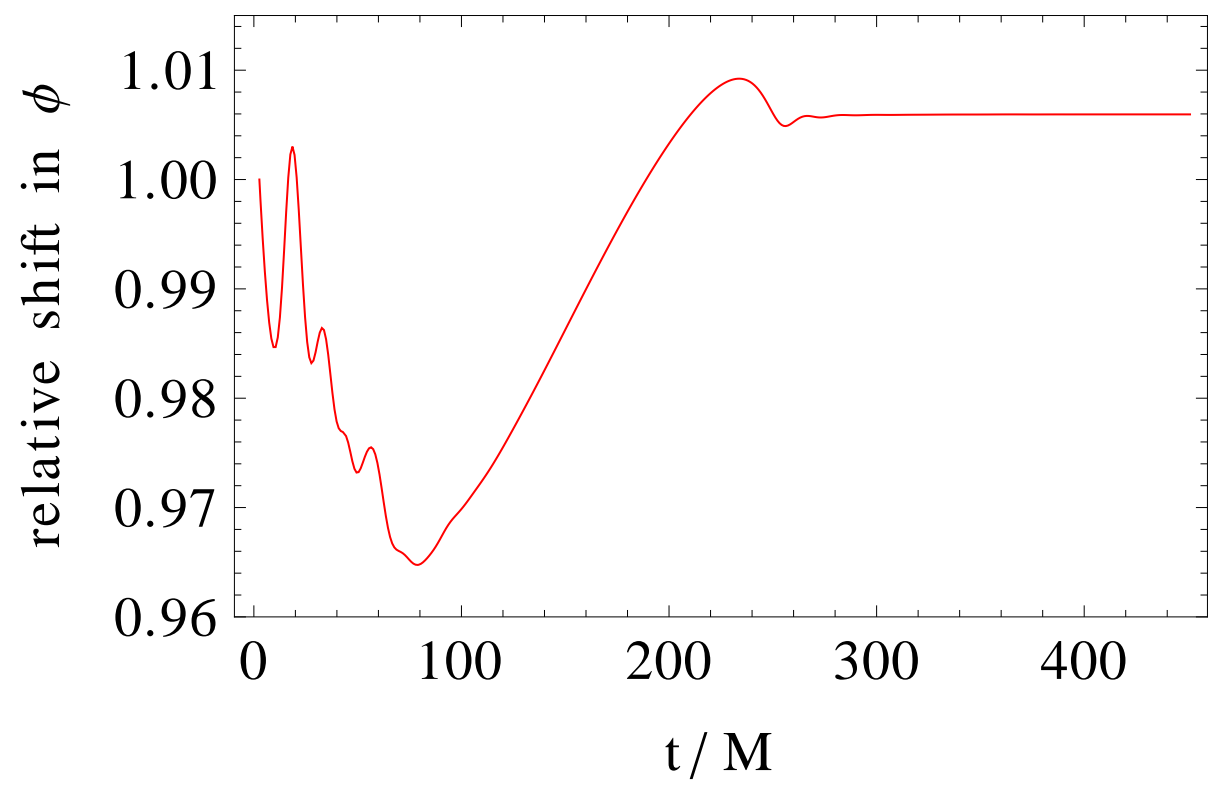

Figure 21: The relative inertial-worldtube $\phi$-coordinate motion of the point circled in Fig. 20 shows approximately a 3.5 percent variation from its initial value.

[17] J. Winicour, "Newtonian gravity on the null cone," J. Math. Phys. 1193 (1983) .

[18] N. T. Bishop, R. Gómez, R. A. Isaacson, L. Lehner, B. Szilágyi, and J. Winicour, 


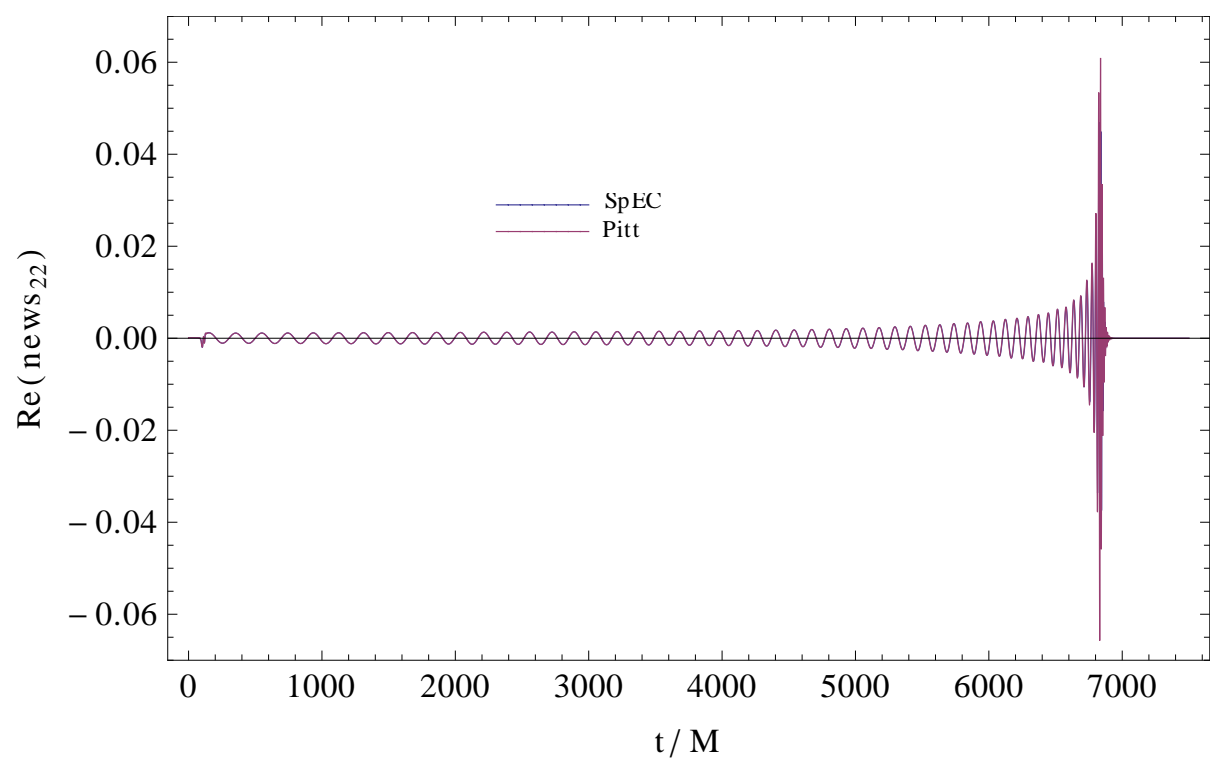

Figure 22: Waveform of the real part of the $(2,2)$ mode of the news function for the generic precessing binary black hole run, showing 26 orbits, inspiral, merger, and ringdown.

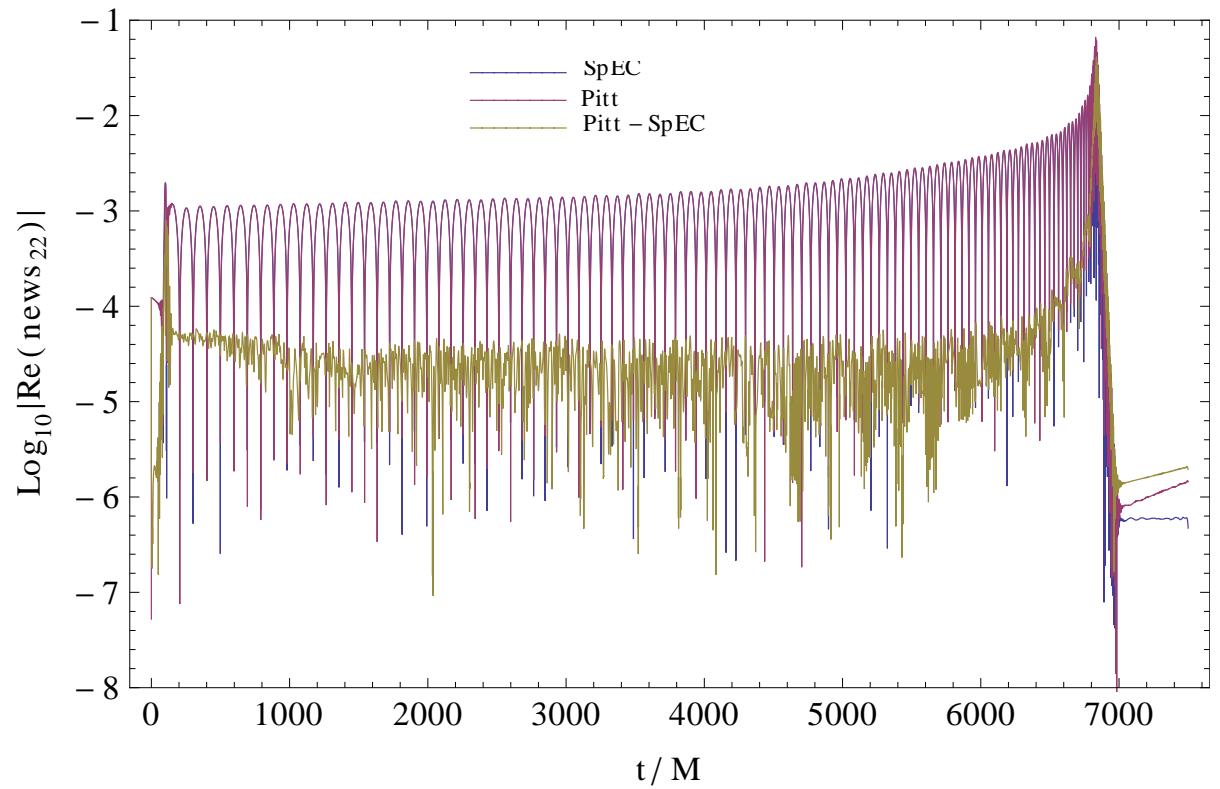

Figure 23: Graphs of $\log _{10} \mid \operatorname{Re}\left(\right.$ news $\left._{22}\right) \mid$ for Pitt and SpEC waveforms for the precessing binary black hole run, showing 16 orbits, inspiral, merger, and ringdown. The difference Pitt - SpEC gives the absolute error $\log _{10}\left|N_{22_{\text {Pitt }}}-N_{22_{S p E C}}\right|$ between the codes, showing consistent agreement throughout the run. 


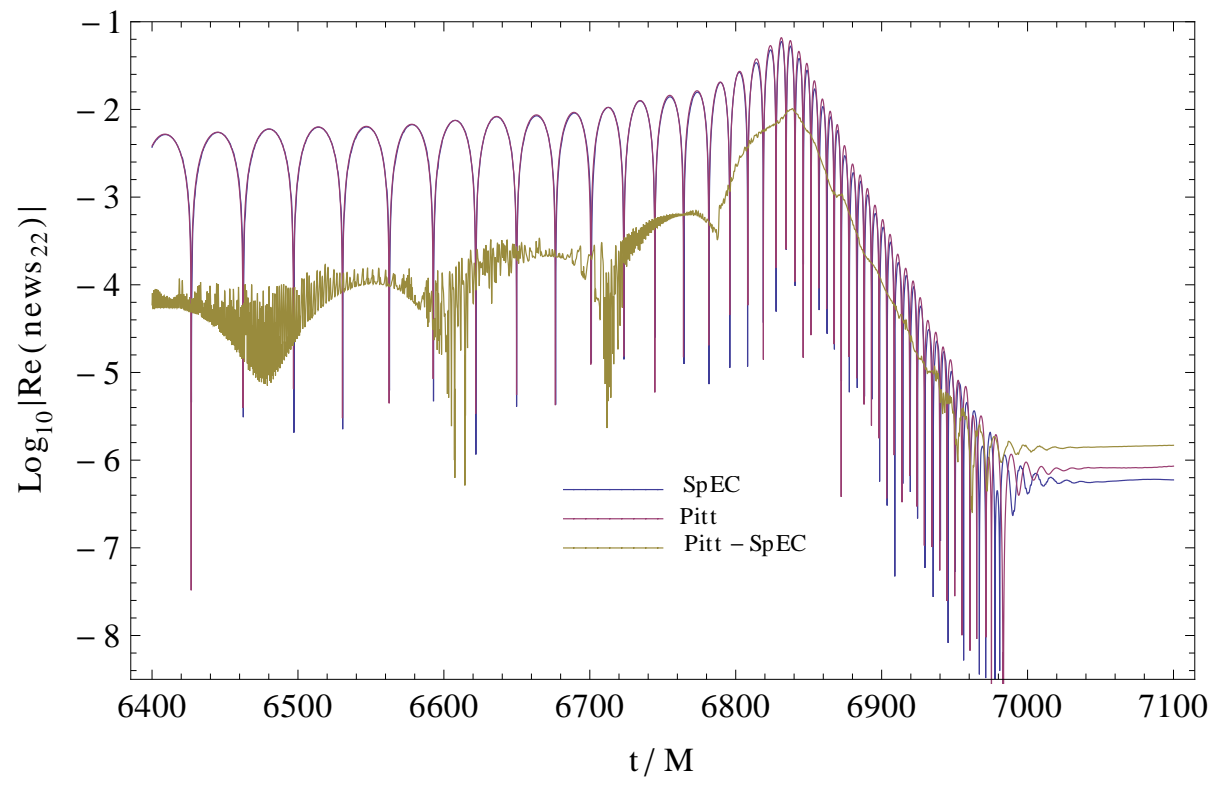

Figure 24: Same graphs as in Fig. 23 but focusing on the merger part of the waveform. Absolute error shows consistency between the Pitt and SpEC news waveforms through merger and ringdown.

"Cauchy-characteristic matching," in Black Holes, Gravitational Radiation and the Universe, B. R. Iyer and B. Bhawal, eds., ch. 24. Kluwer, Dordrecht, 1998. arXiv:gr-qc/9801070.

[19] M. C. Babiuc, N. T. Bishop, B. Szilágyi, and J. Winicour, "Strategies for the characteristic extraction of gravitational waveforms," Phys. Rev. D 79 (2009) 084011, arXiv:0808.0861.

[20] M. C. Babiuc, B. Szilágyi, J. Winicour, and Y. Zlochower, "A characteristic extraction tool for gravitational waveforms," Phys. Rev. D 84 (Aug, 2011) 044057, arXiv:1011.4223 [gr-qc]. http://link.aps.org/doi/10.1103/PhysRevD.84.044057.

[21] E. Newman and R. Penrose, "An approach to gravitational radiation by a method of spin coefficients," J. Math. Phys. 3 no. 3, (1962) 566-578. http://link.aip.org/link/?JMP/3/566/1.

[22] J. Winicour Gen. Rel. and Grav. 19 (1987) 281.

[23] B. Szilágyi, L. Lindblom, and M. A. Scheel, "Simulations of binary black hole mergers using spectral methods," Phys. Rev. D 80 (2009) 124010, arXiv:0909.3557 [gr-qc]

[24] N. W. Taylor, M. Boyle, C. Reisswig, M. A. Scheel, T. Chu, L. E. Kidder, and B. Szilágyi, 


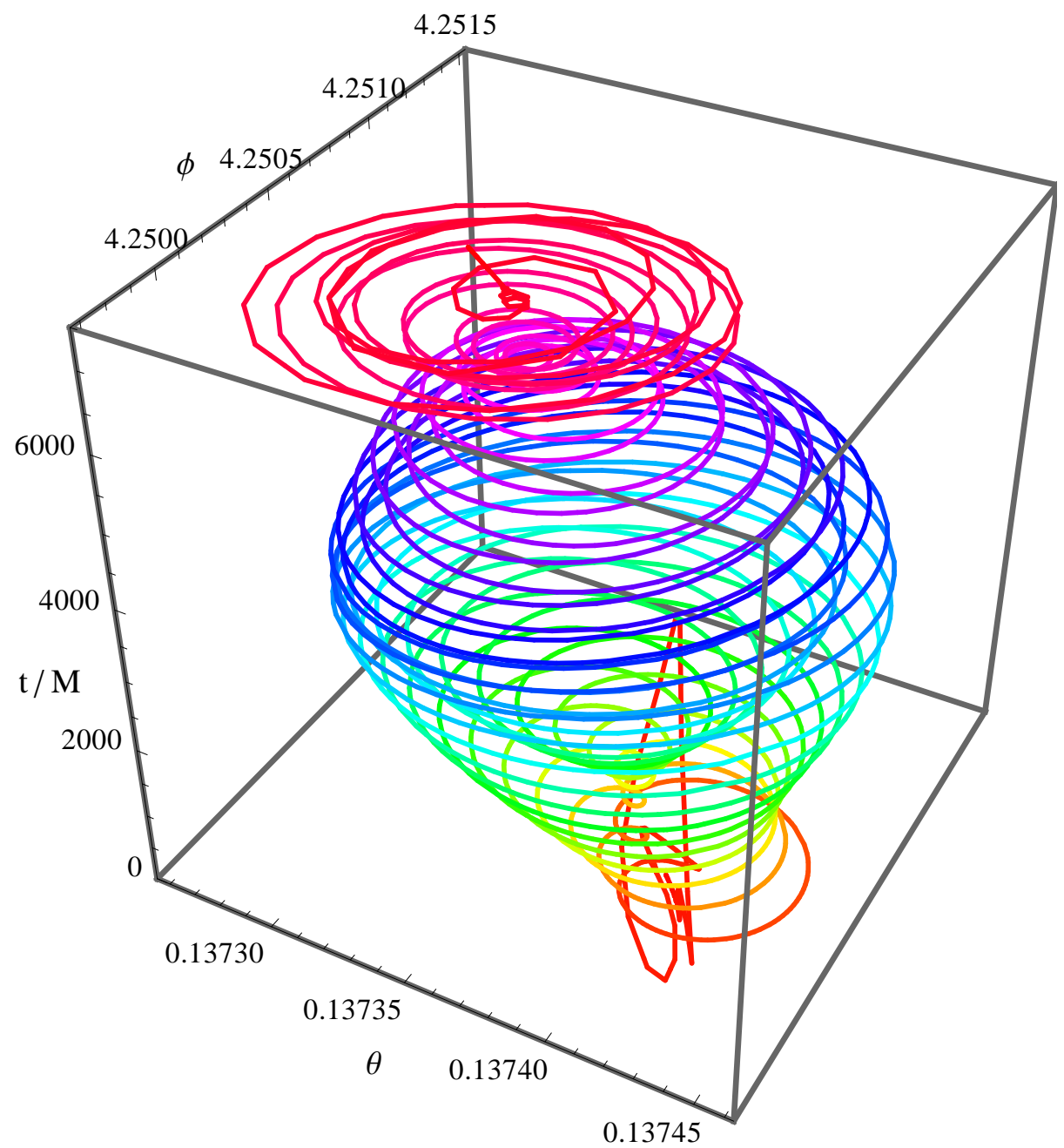

Figure 25: Relative motion of a representative angular coordinate taken from SpEC3 with extraction radius $R=100 M$ showing a long term helical pattern concordant with the black hole inspiral and merger. Globally, initial oscillation due to junk radiation is aligned primarily along the Cartesian $x$ direction, corresponding to the initial black hole orientation. Coordinate motion is an epicyclic helix whose amplitude is modulated by precession of the orbital plane.

"Comparing gravitational waveform extrapolation to Cauchy-characteristic extraction in binary black hole simulations," Phys. Rev. D 88 (Dec, 2013) 124010, arXiv:1309.3605 [gr-qc], http://link.aps.org/doi/10.1103/PhysRevD.88.124010

[25] A. H. Mroue, M. A. Scheel, B. Szilagyi, H. P. Pfeiffer, M. Boyle, D. A. Hemberger, L. E. Kidder, G. Lovelace, S. Ossokine, N. W. Taylor, A. Zenginoglu, L. T. Buchman, T. Chu, 
E. Foley, M. Giesler, R. Owen, and S. A. Teukolsky, "A catalog of 174 binary black hole simulations for gravitational wave astronomy," Phys. Rev. Lett. 111 (2013) 241104, arXiv:1304.6077 [gr-qc]. 